$63 / .84$

$M 14$

RaL History SuRvey of Minnesota

Conway MacMrLlan, State Botanist

\title{
Minnesota
}

\section{Botanical Studies}

\section{Contents}

XVI. On a nex registering balance, Alex. P. Anderson - 177

XVII. On a new electrical auxanometer and continuous recorder, $W . D$. Frost

XVIII. Titles of literature concerning the fixation of free nitrogen by plants, D.T. MacDougal - $\quad-188$

Bulletin No. 9

Purt IV - - Sept. 27,1894

With four plates.

MIN NEA POLIS, MIN N. HARRISON \& SMITH, STATE PRINTERS 1894 


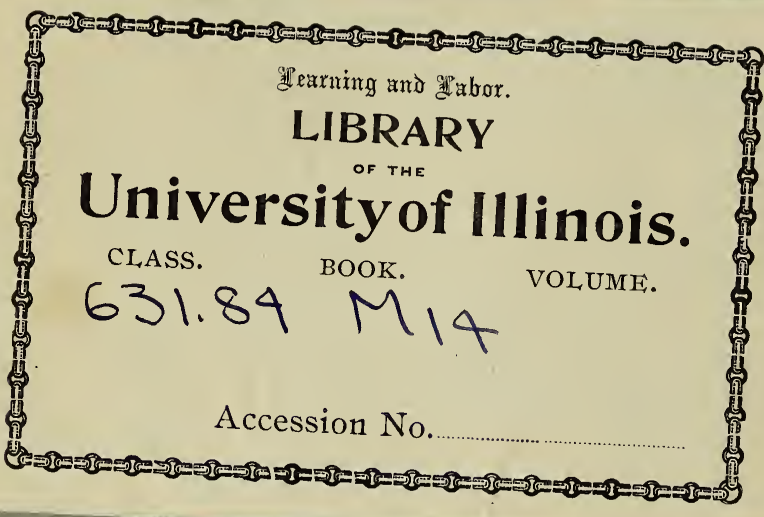




\section{ON A NEW REGISTERING BALANCE.}

Alex. P. Anderson.

In the course of certain experiments on transpiration lately conducted in the laboratories for plant physiology of the University of Minnesota it became apparent that to ascertain the rate of transpiration for some length of time sorne self registering mechanism that could be used to record the increase in the weight of the absorber was necessary. With such an appliance the periodicity (if any) in transpiration could be determined, and a true transpiration curve plotted. After repeated trials and alterations, such a registering balance has been designed consisting essentially of a balance, one arm of the beam of which is lowered by the increase in weight of the calcium chloride absorber.

As this arm is lowered a circuit is closed and an electromagnetic mechanism releases a weight which falls on the other arm of the scale beam, or rather into its scale pan. Thus the scale is balanced automatically, after an increase equal to the weight used has taken place. At the same instant that the weight is released it is recorded on the registering cylinder of the recorder, which can be at any distance from the balance itself. The scale and balancing mechanism are enclosed in a case which entirely protects the whole from falling moisture.

The following detailed description will serve to illustrate the action: The weighing apparatus consists of a platform scale made especially for the purpose, and to fit the registering mechanism. This scale is sensitive to one-fifteenth of a gram, with a capacity of five kilograms. It has a beam eleven inches long, the supports of which are screwed to an iron plate in the bottom of the case, thus making the scale and case practically one and both can be leveled and adjusted together. The brass scale pans are seven inches in diameter and are carried by brass supports, attached to the arms of the scale beam. The scale bearings are of diamond steel.

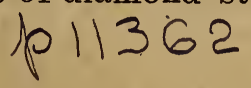


The electro-magnetic balancing mechanism consists of a weight holder and an electro-magnet, together with the contact point on the scale beam, mercury cup, wiring, batteries and the necessary fittings.

The weight holder is a coiled brass tube that holds about one hundred and twenty-five weights. At the lower end of this brass coil is a lever that can turn back and forth on pivot. One end of this lever is connected by a link to the armature of the magnet, and the other end, which is held in place by a spring, when the circuit is open, has a weight pocket that takes one weight from the weight tube each time the circuit is closed and carries it laterally about five-sixteenths of an inch and lets it drop, through a hole in the brass plate, on to the scale pan. As soon as the circuit is opened again by the readjustment of the scale beam the lever returns to its position and receives another weight from the tube, and is again ready to drop it into the scale pan as soon as the necessary increase in weight to close the circuit at the other end of the beam has taken place.

The weight holder has a calibre one-sixteenth of an inch larger than the diameter of the weights used. It is screwed to the frame of the electro-magnet and extends upward and to the outside of the case for the reception of the new weights at its exterior end. It is made air and water tight from the exterior by means of a rubber stopper that fits into the case. The weight holder can therefore be taken out and replaced by one of greater or less calibre, depending upon the size of the weights used, thus if two tubes, five-sixteenths and one-fourth inch respectively, are used, two sets of weights can be put in, viz.: one-fourth inch weighing about one gram, and three-sixteenth inch weighing about one half a gram. Larger or smaller sizes could be used, but for growth or transpiration the above sizes are sufficiently delicate to give a good curve.

The weights used are steel balls, the same make and size as are used in bicycle bearings. These are perfectly accurate, not varying in diameter more than one two thousandths of an inch, and in weight on an average not more than one thousandth of a gram. Sets of these balls can of course be weighed and verified by the experimenter himself.

The electro-magnet has a single coil, and one end of the core is joined with the frame of the magnet which forms the return magnetic circuit. The other end of the core is contracted in the form of a paraboloid. 
The armature having a recess to correspond with this paraboloid, is placed between the two sides of the frame, being pivoted at one end; the other end has a lever communicating with the weight dropping mechanism by a connecting link. This construction of the magnet gives a double magnetic circuit of low resistance, and also a maximum pull and greater range of movement of the armature. The current from a single good carbon-zinc cell is sufficient to operate the weight dropping mechanism. The current from the battery passes through the magnet to a mercury cup, thence through a platinum contact point on the scale beam to the binding post on the case and back to the battery.

The case is twelve by eighteen inches and is made of enamelled sheet iron riveted to a frame work of wrought iron. The edges of the sheet iron are turned in so as to make with the frame work a groove on each side for two sliding glass doors. Thus the whole inside of the registering balance can be seen and watched from without, and either side of the case opened as desired. The case is leveled by means of four milled headed brass screws. A circular spirit level is placed on the iron plate immediately in front of the scale beam supports and thus the entire apparatus can be leveled in a few moments. The whole case is made so that it can be used in a green-house or in the open air without interference from moisture or rain.

The registering balance can be used for registering any continuous increase in weight. For transpiration a combined calcium chloride and sulphuric acid absorber is placed on one scale pan, and the previously dried air that takes up the transpired moisture from the plant chamber (bell glass) is forced through the absorber by means of an aspirator. Two light pieces of rubber tubing connect the absorber with the plant chamber and aspirator, by means of pieces of glass tubing in rubber stoppers fitted into the case. The rubber tubes are thus inside of the case and can not be disturbed by any outward influence. They buoy up and down with the scale pan and absorber. In balancing the scale for the beginning of an experiment these pieces of rubber tubing are partly weighed and continue to be a part of the weight on the absorber pan, but as their weight is approximately constant no error results.

An attachment is made to the balance when used for weighing large fruits, which necessarily must be grown outside of the case. This is made by elongating the scale pan support to 
the exterior by means of a light brass tube. One scale pan will then be on the outside of the case and can be used for the growing fruit.

In the preliminary experiments on transpiration this apparatus has been found to eliminate a large proportion of the errors usually attending this work, while it is of equal value in work on growth increase of weight.

See Plate VII. 


\title{
XVII. ON A NEW ELECTRIC AUXANOMETER AND CONTINUOUS RECORDER.
}

\author{
W. D. Frost.
}

\section{THE ELECTRIC AUXANOMETER.}

In undertaking recently some work on growth in thickness it was found that there was no available auxanometer suitable for the exact needs of the line of experiments designed. Pfeffer's auxanometer was the best instrument within reach of the writer, and while this is adapted for work with moderately large plants it is too cumbersome for delicate ones, as the counter-weight required to overcome the friction of the pulleys is sufficient to produce abnormal conditions. In the measurement of growth in thickness of stems, fruits, etc., it seemed absolutely necessary that the whole instrument used should be attached to, and suspended from the plant, to avoid any error caused by movements, such as twisting or bending, due to heliotropism or geotropism.

To meet these conditions the only contrivance which seemed possible was one in which a very small increment of growth should momentarily close an electric circuit by means of some easily adjustable mechanism. The increment of growth necessary to close the circuit being constant, successive closures of the circuit could, of course, easily be registered.

A working model was constructed upon this principle, and it proved so successful that it has been put in permanent shape by the instrument maker of the laboratory. It has furthermore seemed advisable to print here a description of it in advance of the results from its use in investigations now in progress in the laboratories for plant physiology of the University of Minnesota.

While it was originally intended for measuring growth in thickness, yet it is equally efficient in measuring growth in length. Its extreme lightness and delicacy make it especially useful in measuring the growth of small plants, and since it is 
constructed of aluminum, it can be used on plants while they are under normal conditions of moisture, without injury to the instrument. The auxanometer proper can be separated an indefinite distance from the registering apparatus. Registrations have already been made in the laboratory of the growth of plants under natural conditions, 400 yards distant and in another building.

The auxanometer consists of a ratchet-wheel on a steel axis which also bears a series of small grooved wheels 1 , $3 \frac{1}{2}$ and $6 \mathrm{~mm}$. in diameter, and a somewhat larger wheel upon which is wound a thread bearing a counter-weight. The diameter of the larger wheel, is about $5 \mathrm{~cm}$. and the circumference contains 144 notches. A ratchet which fits in the notches of this wheel, is mounted on an axis similar to that of the others, and has a long horizontal arm. This arm has a platinum tip. As the large wheel turns, the ratchet drops into the notches in its circumference and the platinum tip is lowered so that it touches a drop of mercury which is held in a small cup on the arm of the frame. 'This arm is insulated from the rest of the instrument and is connected by means of a small wire, to one pole of an electric battery. The other part of the instrument is connected with the other pole of the battery. The screw underneath enables the height of the mercury to be regulated, and consequently the length of time which the current remains closed or open.

The frame work of the instrument is made of aluminum, and entire weighs $15 \mathrm{gms}$. It may be attached to the arm of a tripod support, and in this position can be used for measuring growth in length, (as shown in Plate $x$ ). For measuring growth in thickness the instrument can be fastened to the support and held against the stem, or fruit, which is to be measured, or it can be removed from the support and attached to the plant. When attaehed directly it is held in place by a clamp. This can be entirely removed and placed around the plant. It is roughly adjusted in place by means of a catch, which fits into the notches on the clamp. The fine adjustment is accomplished by a screw. When the apparatus is in place a silk thread is fastened to the hook on the frame, passed around the plant in the direction opposite the hands of a clock, so that the thread may be in contact with the entire circumference of the plant. The thread is then passed through a hole in the axis of the auxanometer where it is securely fastened and the 
counter-weight on the wheel is made sufficient to keep taut the thread which passes around the plant.

As the plant increases in thickness the thread is unwound from the pulley upon which it was previously wound; and as this turns, and the teeth of the Jarge wheel pass the ratchet, the electric current is alternately opened and closed.

In measuring growth in length the instrument is supported above the plant and the thread passed from the growing part to the small wheels. If the smallest wheel is used, during the growth of a millimeter, 46 registrations are made, that is to say one-forty-sixth of a $\mathrm{mm}$. in length causes the circuit to be closed, while the largest wheel registers a growth of oneseventh of a millimeter.

See Plate VIII.

\section{THE CONTINUOUS RECORDER.}

This part of the apparatus consists essentially of two rollers, one of which is attached to a clock train, and as it revolves winds upon itself a ribbon of paper on the other roller, and an electro-magnet, to the armature of which is attached a pen that presses against the paper on the second roller. While the circuit remains open a continuous line is traced near one edge of the paper ribbon. When the circuit is closed the pen is drawn to the other side of the paper and the length of the line traced there denotes directly the length of time that the circuit is closed.

The clock train is an eight day lever movement with strong double springs. The case which is seven inches in diameter is finished in brass, and is so attached to the base that it can be easily removed. Projecting through the front of the case is the pinion by which the rollers are turned. This pinion revolves once in twelve hours, carrying with it the roller made of brass carefully turned and balanced. It is slightly less than four inches in diameter; thus the paper moves at a rate of one inch per hour. It has quarter inch flanges, and an arrangement by which the end of the paper is held in place. On the outer surface of this roller is a dial plate with the lettering opposite to that on an ordinary clock. Upon the support is a pointer. By means of this arrangement the time indicated by the clock can be read within a few minutes.

The second roller, with the exception of the dial, is exactly similar to the first. Both are mounted on steel shafts, turned 
to fit the bearings. The supports are made of brass and screwed to a black walnut base, which is built of narrow strips to prevent warping.

The paper ribbon on which the record is obtained is twothirds of an inch in width and made in two lengths, one suffciently long to run four and the other eight days. Across the upper surface of the paper ribbon is printed a series of lines that divide the ribbon into hour spaces, which are numbered consecutively. These hour spaces are so ruled that the time of registration can be read to one minute directly from the ribbon.

A source of error arising from the fact that the paper as it is wound on the first roller increases the circumference of the roller, and causes the paper to move at an increased rate as the paper continues to be wound up, is avoided by having each successive hour-space longer than the preceding one. The correction, however, is very slight on account of the thinness of the paper used, and would amount to only six minutes at the end of the eighth day.

The time marker consists of a pen made of brass, and large enough to hold an amount of aniline ink sufficient for two weeks registration. This pen is attached by means of a rolled brass strip to the armature of an electro-magnet, which is hung on a hinge close to the base. Thus, as the armature moves in response to the attraction of the magnet, or the pull of a tension spring, the pen is drawn through a short horizontal distance. The rod simply serves as a support to the pen. The pen presses against the paper on the roller, and by means of the milled nut it can be kept at any required pressure, or can be withdrawn from the paper entirely when the latter is to be removed or replaced.

When the circuit is open the armature is held back by the tension spring, and the length of the brass strip is so arranged that the pen then traces a line near the right hand side of the ribbon, as it is shown in the plate. When the circuit is closed the armature is attracted and the pen is pushed to the other side of the paper ribbon, thus making a short line at right angles to the length of the paper. If the circuit is immediately opened the time of registration is marked simply by a single cross mark. If, however. the circuit remains closed for some time a line is traced on the left side of the paper.

In reading the record in this case the length of time elapsing between two successive closures of the circuit, is indicated 
by the distance between the two successive forward movements of the pen, or, what is the same thing, the length of the line traced while the circuit is closed, plus the length of the line made while the circuit is open, as any one notch passes the ratchet.

The auxanometer is connected with the registering apparatus and an electric battery. The battery is of a type suited for a closed circuit. The two instruments may be placed upon the same table, or they may be separated any distance, as is most convenient for the operator.

This recorder may also be used with many other kinds of apparatus wherever a continuous record is desired.

See Plate IX. 


\section{TITLES OF LITERATURE CONCERNING THE FIXATION OF FREE NITROGEN BY PLANTS.}

D. T. Mac Dougal.

The relations sustained by plants to the nitrogen compounds of the soil and water, and to the free nitrogen of the air form a subject of great .biological import, and since aside from its purely scientific aspect certain phases of the main question are of vast practical interest they have attracted the attention of the agriculturist and chemist as well as of the botanist.

The results of the investigations, from these various points of view, which have been in progress for a century, form a mass of literature which is scattered through the journals and proceedings of the various branches of natural science in such manner as to be very difficult of access to the student with ordinary facilities.

Among this rich and withal unwieldy mass of literature the part of especial interest to the botanist is that which concerns the fixation of free nitrogen by the leguminous plants and the organism found in the tubercles which characterize this group, and the fixation of free nitrogen by green plants which do not sustain mutualistic relations to the lower organisms.

The large number of controversies resulting from the attainment of radically different conclusions from similar experiments along certain lines of the work, in the hands of various in'restigators, leads to the belief that safe generalizations can be made from the restricted groups of facts thus obtained only when confirmed by extended and parallel researches. To meet this idea the references given below concern the points of central interest to the botanist, beside a number of titles to "nitrification," and to cases of mutualism and symbiosis which may "offer a comparison however distant with the relations existing between the leguminous plant and the tubercle organism. 
The list is composed of titles which have been incidentally collected by Professor MacMillan and the writer, and are comprised in the card catalogue of the botanical department of the University of Minnesota. Their presentation in this form is for the purpose of making them still more readily available to students and investigators in connection with this department, and wherever this line of work is carried forward. It is purposed to bring out a second installment of titles which the writer in the limited time at his disposal was not able to prepare for this number.

Allen E. W. Leguminous plants for green manuring and for $\nmid$ feeding. U. S. Dept. Ag. Farm. Bull. No. 16. 1894.

Alpe and Menozzi. Bull. d. notize agrarie d. Ministere d' Agric. No. 14. 1892.

Andre. See Berthelot.

Arcangeli. Sopra i tubercoli radicali delle Leguminose. Atti del. real. Accad. d. Lincei, 7: Fasc. 6, 223. 1891.

Atkinson G. F. Science Cont. Ala. Ex. Sta. 1:1. 1889.

Atkinson G. F. The genus Frankia in the United States. Bull. Torr. Bot. Club 19: 1711892.

Atkinson G. F. Tubercles of Ceanothus. Bot. Gazette 16: 262.1891.

Atkinson G. F. Contrihutions to the biology of the organism causing leguminous tubercles. Bot. Gazette 18:157, 226, 257. 1893.

Atkinson G. F. Symbiosis in the roots of the Ophioglossaceæ. Bull. Torr. Bot. Club 20:356. 1893.

Atwater and Rockwood. On the loss of nitrogen during germination and growth. Am. Chem. Jour. 8:327. 1868.

Atwater W. 0. On the assimilation of atmospheric nitrogen by plants. Rep. Brit. A. A. S. 685. 1884.

Atwater W. O. Absorption of atmospheric nitrogen by plants. Am. Chem. Jour. 6:365. 1885; also, 12:526. 1891; also, 13:42. 1891 .

$\checkmark$ Atwater and Woods. Atmospheric nitrogen as plant food. Conn. Storrs Ag. Ex. Sta. Rep. 2:11. 1889; also, 3:12. 1890.

Atwater and Woods. The fixation of free nitrogen by plants. Conn. Storrs Ag. Ex. Sta. Rep. 5:17. 1892.

Atwater W. 0. On the liberation of nitrogen from its compounds, and the acquisition of atmospheric nitrogen by plants. Am. Chem. Jour. 8:398. 1868. 
Atwater and Woods. Absorption of atmospheric nitrogen by plants. Am. Chem. Jour. 13:42. 1891.

Babes, Cornil et. Bacteries du sol. Les Bacteries, 1:213. 1890.

Bary A. de Morphologie und Physiologie der Pilze, Flechten. und Myxomyceten. 1866.

Bary A. de Die Erscheinung der Symbiose. 1879.

Benecke F. Ueber die Mykorhiza. Biol. Centralb. 4:753, 781. 1888.

Benecke F. Ueber die Knöllchen an den Leguminosen-Wurzeln. Bot. Centralb. 29:53. 1887.

Berg F. Das nitrificierende Ferment des Bodens. Sitzungsb. Naturf. Ges. Dorpät. 10: No. 1. 1892.

Berggren S. Om rotbildningen hos australa Coniferer. Bot. Notiser. 144. 1887.

Berthelot. Sur l'absorption de l'azote libre par les principes. immédiats des végétaux sous l'influence de l'electricité. Jour. f. Pharm. 24:433. 1876.

Berthelot. Sur l'absorption de l'azote libre par les principes immédiats des végétaux sous l'influence de l'electricité atmospherique. Compt. rend. 83:677. 1876.

Berthelot. Sur l'absorption de l'azote libre par les principes. immédiats des végétaux sous l'influence de l'electricité. Ann. d. Chim. et Phys. 10:55. 1877.

Berthelot and Andre. "Nitrates in plants." Jour. f. Pharm. et Chim. 1884.

Berthelot. Fixation directe de l'azote atmospherique libre par certaines terrains argileux. Compt. rend. 101: 1885.

Berthelot and Andre. Sur les principles azoteés de la terre végétale. Compt. rend. 103:1101. 1886.

Berthelot. Sur la fixation directe de l'azote gazeux de l'atmosphere par les terres végétales. Compt. rend. 104:205. 1887.

Berthelot. Sur la fixation de l'azote gazeux de l'atmosphere par les terres végétales, avec le concours de la végétation. Compt. rend. 104: 625. 1887.

Berthelot. Experiences nouvelles sur la fixation de l'azote et par certaines terres végétales et par certaines plantes. Compt. rend. 106:372. 1888.

Berthelot. Sur quelques conditions generales de la fixation de l'azote par la terre végétale. Compt. rend. 106:569. 1888. 
Berthelot. Sur la transformation dans le sol des azotates en composes organiques azotes. Compt. rend. 106:638. 1888.

Berthelot. Observations sur la fixation de l'azote par certaines sols et terres végétales. Compt. rend. 106:1049. 1888.

Berthelot. Sur la fixation de l'azote par la terre végétale. Compt. rend. 106:1214. 1888.

Berthelot. Experiences nouvelles sur la fixation de l'azote par. certaines terres végétales et par certaines plantes. Ann. d. Chim. et Phys. 16. 1889.

Berthelot and Andre. Faits pour servir à l'histoire des principes azotes renfermes dans la terre végétale. Ann. $d$. Chim. et Phys. 6 ser. 25:314. 1892.

Berthelot. Sur la fixation de l'azote dans les oxydation lentes. Compt. rend. 108:543. 1889.

Berthelot. Fixation de l'azote par la terre végétale nue ou avec le concours des Leguminéuses. Compt. rend. 108: 700. 1889.

Berthelot. Recherches nouvelles sur la fixation de l'azote par la terre végétale. Influence del electricité. Compt. rend. 109:281. 1889.

Berthelot. Sur les relations de l'azote atmospherique avec la terre végétale. Compt. rend. 109:345. 1889.

Berthelot. Sur la fixation de l'azote atmospherique. Compt. rend. 109:417. 1889.

Berthelot. Observations sur la formation de l'ammoniaque et de composes azotes volatils aux dépens de la terre végétale et des plantes. Compt. rend. 109:419. 1889.

Berthelot. Remarques sur la formation des azotates dans les végétaux. Compt. rend. 110:109. 1890.

Berthelot. Observations sur la note precedente de Schloesing fils et Em. Laurent. Compt. rend. 111:753. 1890.

Berthelot. Observations sur les reactions entre la terre végétale et l'ammoniaque atmospherique. Compt. rend. 111:558. 1890.

Berthelot and Andre. Faits pour servir a l'histoire des principes azotes renfermes dans la terre végétale. Compt. rend. 112:189. 1891.

Berthelot. Nouvelles recherches sur la fixation de l'azote par les microbes. Compt. rend. 115:569. 1892.

Berthelot. Recherches nouvelles sur les micro-organismes fixateurs de l'azote. Compt. rend, 116:842. 1893, 
Berthelot. Observations relativus a une note de Winogradsky Sur l'assimilation de l'azote gazeux de l'atmosphere par les microbes. Compt. rend. 116:1388. 1893.

Berthelot. Nouvelles recherches sur les micro-organismes fixateurs de l'azote. Ann. d. Chim, et Phys, 30:419. 1893.

Berthelot. Nouvelles recherches sur la fixation de l'azote atmospherique par les micro-organismes. Ann. d. Chim. et Phys. 30:411. 1893.

Berthelot. Sur ure methode destinee a étudier les echanges gazeux entre les étres vivants et l'atmosphere que les entoure. Compt. rend. 118:112. 1894.

Berthelot. Nouvelles observations sur les composes azotes volatils emis par la terre végétale. Compt. rend. 118: 195,1894 .

Beyerinck M. W. Die Bacterien der Papilionaceen-knöllchen. Bot. Ztg. 46: 725, 741, 757, 780, 797. 1888.

Beyerinck M. W. Wurzelknospen. Verh. d. koninkl. Akad v. Wetensch. te Amsterdam. 25:-. 1887. See also 1890.

Beyerinck M. W. Künstliche Infektion von Vicia faba mit Bacillus radicicola: Ernährungsbedingungen dieser Bacterie. Bot. Ztg. 48:838. 1890.

Beyerinck M. W. Over ophooping van atmospherische Stickstoff in culturen van Bacillus radicicola. Vers. en Meded. der koninkl. Akad. van Wetensch. te Amsterdam. Afd. Natuurkunde, Hft. 3:460. 1891.

Böhme K. Stickstoff-ernährung der Leguminosen. Separat 1892. Dresden.

Bolley H. B. Notes on root tubercles of indigenous and exotic Legumes in virgin soil of Northwest. Ag. Science $7: 58.1893$.

Bonnier G. La Constitution des Lichens. Jour. d. Bot. 1:1. 1887.

Bonnier G. Germination des spores de Lichens sur les protonèmas des Mousses. Rev. Gen. d. Bot. 1:165. 1889.

Bonnier G. Recherches sur la synthese des Lichens. Ann. d. Sc. Nat 7 ser. 9 :1. 1889.

Bonordion H. F. Handbuch der Allgemeinen Mykologie. 1851.

Bordier M. H. "Nitrification." Mem. Sc. Phys. et Nat. Bordeaux 5:185. 1890.

Borgreve. Zusatz zu: Braun zur Mycorhiza Frage. Forstl. Bl. 13:205. 1889 . 
Bouche. Zur Unterscheidung des Phaseolus vulgaris und $\mathrm{Ph}$. multiflorus Lam. Bot. Ztg. 10:735. 1852.

Boudier. $\mathrm{Du}$ parasitisme probable de quelques espèces du genre Elaphomyces et de la recherche de ces Tubéracés. Bull. d. Soc. Bot. d. France, 23:-. 1876.

- Bouquet. Eine neue Hypotheses des Absorptions der Stickstoff durch Pflanzen. Jour. l'Ag. Practique. 1888.

Boussingault J. B. Recherches chimiques sur la végétation enteprises dans le but d'examiner si les plantes prennent de l'azote de l'atmosphere. Ann. Sc. Nat. 10:257. 1837.

Boussingault J. B. Recherches chimiques sur la végétation enterprises dans le but d'examiner si les plantes prennent de l'azote de l'atmosphere, Compt. rend. 6:102. 1838 and $\boldsymbol{\gamma}: 138.1838$.

Boussingault J. B. Recherches chimiques sur la végétation enteprises dans le but d'examiner si les plantes prennent de l'azote de l'atmosphere, Ann. d. Chim. et Phys. 67:35. 1838 and 69:353. 1838 .

Boussingault J. B. Recherches chimiques sur la végétation enteprises dans le but d'examiner si les plantes prennent de l'azote de l'atmosphere, Jour. f. Prakt. Chem. 14:193. 1838, and 16:385. 1839 .

Boussingault J. B. Recherches sur la végétation, etc. Ann. d. Chim. 41:5. 1854, and 43:149. 1855.

Boussingault J. B. Recherches sur la végétation, etc. Ann. Sc. Nat. 1: 241.1854.

Boussingault J. B. Recherches sur la végétation, etc. Jour. d. Pharm. 26:127. 1854.

Boussingault J. B. Recherches sur la végétation. Compt. rend. 38: 580, 607. 1854, and 39:601. 1854.

Boussingault J. B. Recherches sur la végétation. Ann. d. Chim. 46:5. 1856.

Boussingault J. B. Recherches sur la végétation. Ann. Sc. Nat. 2:357. 1854.

Boussingault J. B. Recherches sur la végétation. Jour f. Prakt. Chem. 62:108, 181. 1854, and 63:418. 1854.

Boussingault J. B. Recherches sur l'influence que l'azote assimilable des engrais exerce sur la production de la matiere vegetale. Compt. rend. 44:940. 1857, and 45: 833. 1857. 
Boussingault J. B. Recherches sur l'influence que l'azote assimilable des engrais exerce sur la production de la matiere vegetale. Ann. Sc. Nat. 7: 5. 1857.

Boussingault J. B. Agronomie, Chemie, Agricole et Physiologie 1:198. 1860, and 2:340. 1861.

Boussingault J. B. Sur la nitrification de la terre végétale. Compt. rend. 7 6: 22 . 1873.

Boussingault J. B. Sur la nitrification de la terre végétale. Ann. d. Chim. et Phys. 29:186. 1873.

Boussingault J. B. Sur l'influence que la terre végétale exerce sur la nitrification des substances azotes d'origine organique employees comme engrais. Compt. rend. 82:477. 1876. Also, Ann. d. Chim. 8:5. 1876.

Boutin. Note sur l'origine des nitrates dans l'Amarantus blitum. Compt. rend. 82: No. 25. 1876.

Boutroux L. Sur la fermentation panaire. Compt. rend. 112: 203. 1891. See also Ann. d. Chim. et Phys. 1892.

Boutroux L. Reveux des travaux sur les bacteries et les fermentations. Rev. Gen. d. Bot. 16:30. 1894.

Bowman J. E. On the parasitical connection of Lathraea squamaria and the peculiar structure of the subterranean leaves. Trans. Linn. Soc. 16:399. 1833.

Braun. Zur Mycorhiza Frage. Forstl. Bl. 13:204. 1889.

$\checkmark$ Breal E. Ann. Agron. 1888.

Breal E. Observations sur la fixation de l'azote atmospherique par les Légumineuses dont les racines portent des nodosités. Compt. rend. 107:372. 1888.

Breal E. Fixation de l'azote par les Légumineuses. Compt. rend. 109:670. 1889. 110:670. 1890.

Breal E. Bindung des Luftstickstoffs durch Kresse (Tropaeolum). Ann. Agron. 18:369. 1893.

Brefeld 0. Bot. Untersuchungen. 1883.

Brefeld 0. Untersuchungen auz dem Gesammtgebiete der Mykologie, 3. 1877. And \%. 1888.

Bruchmann H. Ueber Anlage und Wachstum der Wurzeln von Lycopodium und Isoetes. Jen. Zeitschr. f. Naturw. New Ser. 1:522. 1874.

Bruchmann H. Die Dichotomie der Wurzel von Pinus sylvestris. Jen. Zeitschr. f. Naturw. New Ser. 1:572. 1874.

Bruchmann H. Das Prothallium von Lycopodium. Bot. Centralb. 21:23, 309. 1885 . 
Brunchorst B. Ueber die Knöllchen an der W Wurzeln von Alnus und den Eleagnaceen. Bot. Centra lb. 24:222. 1885.

Brunchorst B. Ueber die Knöllchen an den Leguminosenwurzeln. Ber. d. deut. Bot. Ges. 3: Hft. 7, 241. 1885.

Brunchorst B. Ueber einige Wurzelanschwellungen, besonders diejenigen von Alnus and Elaeagnaceen. Untersuch. Bot. Inst. z. Tubingen, 2:151. 1886.

Brunchorst B. Die Struktur der Inhaltskörper in den Zellen einiger Wurzelanschwellungen. Sep. a. Bergens Mus. Arsberetning. 235. 1887.

Buscalioni, Si contengono bacteri nei tubercoli radicati delle Leguminose. Malphigia 1:21, 464. 1887.

Cloez S. Recherches experimentales sur la nitrification et sur la source de l'azote dans les plantes. Compt. rend 41:935. 1855 .

Cloez S. Lettre a M. Chevreul concernant les experiences de M. Ville sur la question de l'assimilation de l'azote de l'air par les végétaux. Compt. rend. 41:775. 1855.

Cloez S. Recherches experimentales sur la nitrification et sur la source de l'azote dans les plantes. L'Institute $\mathbf{2 3}$ : 417. 1855 .

Cloez S. Recherches experimentales sur la nitrification et sur la source de l'azote dans les plantes. Jour. f. Prakt. Chem. 68:138. 1856.

Cloez S. See Gratiolet.

Clos D. Ebauche de la rhizotaxie 1848. Paris.

Clos D. Du collet dans les plantes et de la nature de quelque tubercles. Ann. d. Sc. Bot. 3: 13.1849.

v Clos D. Du collet dans les plantes et de la nature de quelques tubercules. Ann. Sc. Nat. 13:5. 1850. See also Soc. Philom. Proc. Verb. 34. 1850.

LClos D. Revision des tubercles des plantes et des tuberculoides des Légumineuses, Mem. d. l'Acad. d. Toulouse 9 ser. 5. 1.1893.

Canevari A. La nutrizione delle piante il terreno e la concimazione, L'Italia agricolia 19.1887.

-Chevreul E. Sur le role de l'azote atmosphérique dans l'economie végétale. Compt. rend. 107:1460. 1888.

Chuard E. Sur l' existence de phénoménes de nitrification dans les mileux riches en substance organiques et a reaction acids. Compt. rend. 114:181. 1892. 
Cohn F. Ueber parasitic Algen. Beitr. z. Biol. 1: Hft. 2:87. 1872.

Cohn F. Zur Geschichte der Leguminosenknöllchen. Centralb. f. Bakt. u. Par-Kunde. 10: No 6, 190. 1891.

Coignet J. De l'absorption de l'azote par les végétaux. Ann. d. 1. Soc. d'agr. d. Lyon, 5 Ser. 2:233. 1888.

Condamy A. Etude sur l'histoire naturelle de la Truffe. 1876. Conn H. W. Nature of the root tubercles of leguminous plants. U. S. Ex. Sta. Rec. 2:686. 1890 and 1891, and $3:$ : $6 . \quad 1891$ and 1892.

Conn H. W. Free nitrogen assimilation. Bull. Torr. Bot. Club 20: 148. 1893.

Corda A. K. J. Icones Fungorum hucusque cognitorum. 1837, 1854.

Cornil et Babes. Bacteries du sol. Les Bacteries 1:213. 1890.

Cornu. Commission du Phylloxéra. 1876.

Cornu. Etudes sur le Phylloxéra vastatrix. 1878.

Coudon Muntz. and La fermentation ammoniacale de la terre. Ann. Agron. 19: 209.1893.

Crochetelle et Dumont. Sur la nitrification des terre de prairie, Compt. rend. 117:670. 1893.

Langeard. Note sur les Mycorhizes endtropiques. Le Botaniste Ser. 2, 223. 1891;

De Candolle. Prodromus Syst. Nat. Reg. Veg. 2:312. 1825.

Deherain P. P. Sur l'absorption de l'azote par les plantes. Bull. Soc. Chim. 16:2. 1871.

Deherain P. P. Sur l'intervention de l'azote atmospherique dans la vegetation. Compt. rend. 73:1352. 1891. And $76: 1390.1873$.

Deherain P. P. Sur l'intervention de l'azote atmospherique dans la vegetation Bull. Soc. Chim. 19:538. 1873.

Deherain P. P. Sur l'intervention de l'azote atmospherique dans la vegetation. Jour. d. Pharm. 18:95. 1873.

Deherain P. P. Sur l'intervention de l'azote atmospherique dans la vegetation. Ann. Sci. Nat. 5 Ser. 18:147. 1873.

Deherain P. P. Sur l'intervention de l'azote atmospherique dans la vegetation. Chem. News. 29:271. 1874.

Deherain P. P. Sur l'enrichessement en azote d'un sol maintenu en prairie. Compt. rend. 101. 1885.

Deherain P. P. Le travail du sol et la nitrification. Ann Agron. 19:401. 1893. 
Deherain P. P. Le travail de la terre et la nitrification. Compt. rend. 116:1091. 1893.

Delpino. Osservazioni sopra batteriocecidii la sorgente l'azote in una piante-Galega officinalis. Malphigia 2:385. 1888.

De Saussure. Recherches sur la vegetation. 206. 1804. Detmer. Physiol. Chem. Unters. uber Keimung 68. 1875.

Devaux. Circulation passive de l'azote dans les végétaux. Jour. d. Bot. 5:130. 1891.

Devaux. Les echanges gazeux d'un tubercle, representes schematiquement par un appareil physique, Bull. Soc. Bot. d. France. 37:257. 1891.

Devaux. Atmosphere interne des tubercules et racines tuberculeuses. Bull. d. Soc. Bot. d. France. 37:272. 1890.

De Vogue. Fixation de l'azote ammoniacal sur la paille. Compt. rend. 115: 25. 1892.

De Vries. Wachstumgeschichte des roten Klees. Landw. Jahrb. 6: 933.1877.

Douliot et van Tieghem. Recherches comparatives sur l'origine des membres endogenes dans les plantes vasculaires. Ann. d. Sc. Nat. 7 Ser. 8:1. 1888.

Drouin. See Gautier.

Duchartre P. Note sur l'Hypopitys multiflora Scop. Ann. d. Sc. Nat. 3 Ser. 6: 29.1846.

Duclaux. La distribution de la matiere organique et des microbes dans le sol. Ann. Inst. Past. 7:823. 1893.

Duhamel du Monceau. La Physique des Arbres. 1758.

Dumont et Crochetelle. Sur la nitrification des terres de prairie. Compt. rend. 117:670. 1893.

Drobnig. Beitrage zur Kenntniss der Wurzelknöllen. Inaug. Diss. 1892. Röstock.

Drude 0. Die Biologie von Monotropa Hypopitys L. und Neottia Nidus avis L. 1873. Göttingen.

Ehrenberg A. Weitere Untersuchungen üeber die Frage nach dem Freiwerden von gasformigen Stickstoff bei Faulniss-processen. Zeitschr. f. phys. Chem. 11 : Hft. 5. 1887.

LEidam E. Ueber Pilzenentwickelung in den Wurzeln der Orchidaceen. Jahresber. d. schles. Ges. f. vaterl. Cultur. 57:297. 1879.

Emmerling A. Untersuchungen über die Stickstoffernährung der Pflanzen. Mitt. a. d. Lab. d. Versuch. Sta. i. Kiel. Hft. 1. 1879. 
Emmerling A. Studien über die Eiweissbildung in der Pflanze. Landw. Vers. Sta. Abh. 1. 1879.

Eriksson J. Studier öfver Luguminosernas rötknoler. Doctordiss. Lund. 1874.

Also, Acta Univ. Lund. 10. 1873.

Farlow. Tubercles on Leguminous Roots. Garden and Forest $1: 135.1888$.

Fink K. Die Mikroorganismen in der Ackerkrume. Fühling's Landw. Ztg. 43: 48, 853. 1893.

-Fitz A. Stickstoffquelle fur Mucor. Deut. Chem. Rev. 8:1540. 1876.

Fisch Rees. und Untersuchungen über den Bau und Lebensgeschichte der Hirschtruffel-Elaphomyces. Biblioth. Bot. Hft. 7.1. 1887.

Frank. Ueber die biologische Verhältnisse des Thallus einiger Krustenflechten. Cohn's Beitr. z. Biol. d Pflanzen. 2:123. 1876.

Frank. Ueber die Parasiten in den Wurzelanschwellungen der Leguminosen. Bot. Ztg. 37: 377, 393. 1879.

Frank. Handbuch der Pflanzenkrankheiten. 131, 647, 674, 748.1880.

Frank. Ueber die auf Wurzelsymbiose beruhende Ernährung gewisser Bäume durch unterirdische Pilze. Ber. d, deut. Bot. Ges. 3: 128.1885.

Frank. Neue Mittheilungen ñber die Mycorhiza der Bäume und der Monotropa hypopitys. Ber. d. deut. Bot. Ges. 3:- 1885.

Frank. Mikroorganismen des Erdbodens Ber, d. deut. Bot. Ges. 4: Hft. 8. 1886.

Frank. Ueber die Quellung der Stickstoffnahrung der Pflanzen. Ber. d. deut. Bot. Ges. 4:293. 1886.

Frank. Sind die Wurzelanschwellungen der Erlen und Elaeagnaceen Pilzgallen? Ber. d. deut. Bot. Ges. 5:50. 1887.

Frank. Ueber neue Mykorhiza Formen. Ber. d. deut. Bot. Ges. 5:395. 1887.

$\checkmark$ Frank. Ursprung und Schicksal der Saltpetersaure in der Pflanze. Ber. d. deut. Bot. Ges. 5.472. 1887.

Frank. Ernährung der Pflanzen mit Stickstoff, und ueber den Kreislauf desselben in der Landwirtschaft. Sep. 1888. Berlin. 
Frank. Ueber den Einfluss welche das Sterilisiren des Erdboden auf die Pflanzenentwickelung ausübt. Ber. $d$. deut. Bot. Ges. 6:- - 1888.

-Frank. Ueber die physiologische Bedeutung der Mycorhiza. Ber. d. deut. Bot. Ges. 6:248. 1888.

Frank. Untersuchungen über die Ernährung der Pflanze mit Stickstoff. Landw. Jahrb. 18:419, 496. 1888.

rFrank. Was nützen den Waldbäumen die Wurzelpilze. Forst. Bl. Jan. 1. 1889.

Frank. Ueber den Experimental Nachweis der Assimilation freien Stickstoffs durch erdboden bewahnenden Algen. Ber. d. deut. Bot. Ges. 7:34. 1889.

Frank. Ueber die gegenwartige Stamm unserer Kenntnisse der Assimilation elementaren Stickstoffs durch die Pflanze. Ber. d. deut. Bot. Ges. 7:234. 1889.

Frank. Pilzsymbiose der Leguminosen. Sep. 1890. Berlin. Frank. Pflanzenphysiologie, 120. 1890. Berlin.

Frank. Ueber Assimilation von Stickstoff aus Luft durch Robinia Pseudacacia. Ber. d. deut. Bot. Ges. 8: 292. 1890.

Frank und 0tto. Untersuchungen uber Stickstoffsassimilation. in der Pflanze. Ber. d. deut. Bot. Ges. 8:331. 1890.

Frank und 0tto. Untersuchungen über Stickstoffassimilation in der Pflanze. Naturw. Wochen Schr. 6:215. 1891.

-Frank. 'Neue Stimmen über die Stickstofffrage. Deut. Landw. Presse, 2 1:119. 1894.

Frank. Inwieweit ist der freie Luft-Stickstoff für den Ernährung der Pflanzen verwerthbar? Deut. Landw. Presse, 18:779. 1891.

Frank. Ueber einege neue Versuche betreffs der Stickstoffsassimilation in der Pflanze. Deut. Landw. Presse. 18:403. 1891.

Frank. Ueber die auf Verdauung von Pilze abzielende Symbiose der mit endotropic Mykorhizen begabten Pflanzen sowie, der Leguminosen und Erlen. Ber. d. deut. Bot. Ges. 9:244. 1891.

Frank. Ueber die auf den Gasaustausch bezüglichen Einrichtungen und Thätigkeiten der Wurzelknöllchen der Leguminosen. Ber. d. deut. Bot. Ges. 10:271. 1892.

Frank. Lehrbuch der Botanik 1:574. 1892. 
Frank. Die Assimilation freien Stickstoffs bei den Pfanzen in ihrer Abhängigkeit von Species, von ErnhärungsVerhaltnissen und von Bodenarten. Landw. Jahrb. 21: Hft. 1 and 2.1892.

Frank. Noch ein Wort zur Stickstoff-Frage. Deut. Landw. Presse, 20: 183.1893.

Frank. Die Assimilation des freien Stickstoffs durch die Pflanzenwelt. Bot. Ztg. 51: Abth 1. 138. 1893.

Frank. Sur l'importance des Mycorhizes dans la nutrition des plantes humicoles. Rev. Eaux et Forets 38:122. 1894.

Frank. Die Bedeutung der Mycorhiza die gemeine Kiefer. Forstwiss. Centralb. April. 1894.

Frankland E. Chemical changes in their relations to Microorganisms. Rep. Brit. A. A. S. 681. 1884.

Frankland P. and G. C. The nitrifying process and its specific ferment. Proc. Roy. Soc. Lond. 47:296. 1890. Phil. Trans. Roy. Soc. 181:107. 1891.

Frankland P. Micro-organisms in their relation to chemical change. Nature 46:135. 1892.

Fries E. Sclerotium. Systema Mycologicae 2:250. 1823.

Fries Th. M. Om Vaxtbolag. Sartryck ur Svensk Tidskrift. 1892.

Fromberg P. F. H. On the source of carbon and nitrogen in plants as derived from the soil. Jour. Ag. 2:41. 1847.

Gain. Influence de l'humidité sur le development des nodosités des Leguminosae. Compt. rend. 116: 1394. 1893.

Gasparini G. Osservazioni sulla struttura dei spongiolari di alcune piante leguminose. Lette all. Ac. di. Napolli. 1851.

Gasparini G. Richercha sulla natura dei succiatoria la escrezione delle radici. 1856.

Gautier et Drouin. Recherches sur la fixation de l'azote par le sol et les végétaux. Compt. rend. 106: 754, 863, 944, 1098, 1174, 1232, 1460, 1605.1888.

Gautier und Drouin. Fixirung von Stickstoff durch den Boden und die Gewasser. Chem. Centralb. 1: No. 8. 1892.

Gautier et Drouin. Remarques sur le mecanisme de la fixation de l'azote par le sol et les vegetaux. Compt, rend. 114: 19.1892. 
Mac Dougal: FIXATION OF FREE NITROGEN.

Gautier und Drouin. Fixirung des atmosphäreschen Stickstoffes durch Boden und Pflanze. Chem. Centralb. 1: No. 11. 1892.

Gibelli G. La malattia del castagno, ozzervazioni esperiene. 1879.

Gibelli G. Nouvelles etudes sur la maladie du Chataignier dite maladie de l'encre. Arch. d. Biol. Ital. 3:122.

Gibelli G. Nuova studi sulla malattia del castagno detta dell' inchiostro. 1883. Bologna.

Gilbert. See Laws.

Gilbert. Einige neue Ergebnisse betr. d. Stickstoff Quelle d. Pflanze. Chem. Centralb. No. 46. 1886.

Godlewski E. Zur Kenntniss der Nitrification. Anzeig. Akad. Wiss. in Krakau. 1892.

Goebel K. Ueber Prothallium und Keimpflanzen von Lycopodium inundatum. Bot. Ztg. 45:161. 1887.

Goebel K. Zur Keimungsgeschichte der Farne. Ann. d. Jard. Bot. d. Buitenzorg. 7: 74 . 1888.

Goebel K. Morphologische und Biologische Studien. 4. Ueber Javanische Lebermoose. Ann. d. Jardin. Bot. d. Buitenzorg 9:6. 1891.

Gottsche C. M. Anatomischphysiologische Untersuchungen über Haplomitrium Hookeri. Verh. d. kais. Leop. Car. Akad. d. Naturf. 12: Abth. 1. 265. 1843.

Gottsche C. M. Ueber das Genus Monoclea. Bot. Ztg. 15:281. 1857.

Gottsche C. M. Uebersicht und kritische Wurdigung der seit dem Erscheinen der Synopsis Hepoticorum bekannt gewordenen Leistrungen in der Hepoticologie. Bot. Ztg. Beilage 16:140. 1858.

Gratiolet et Cloez. Recherches experimentales sur la végétation des plantes, submergees. Ann. d. Chim. et Phys. 32: 41 . 1851.

Gratiolet et Cloez. Recherches experimentales sur la végétation des plantes submergees. Jour. f. Prakt. Chem. 52: 275 . 1851, and 53: 181.1851.

Gravis A. Observationes anatomiques sur les excroissances des racines de l'aume. Bull. d. 1. Soc. d. Bot. d. Belgique. 18: Pt. 1. 50.1879.

Gravis A. Note sur les excroissances des racines de l'aune. Compt. rend. d. l. seance men. d. 1. Soc. roy. d. bot. Belgique. 1880. 
Gregory E. Notes on some botanical reading done in the laboratory of Professor Schwendener in Berlin, in June and July, 1889. Bull. Torr. Bot. Club 16:297. 1889.

Grosglik S. Die Mycorhiza. Wszechswiat 4:725. 1885. Warschau.

Halsted B. D. Nitrogen assimilation in clover: A hint. Bull. Bot. Dept. Ag. Coll. Iowa. Feb., 1888.

Hariot P. Sur une Algue qui vit dans les racine d. Cycad. Compt. rend. 115: 325.1892.

Hartig R. Ueber die symbiotische Erscheinungen im Pflanzenleben. Bot. Centralb. 25:350. 1886.

Hartig R. Die pflanzliche Wurzelparasiten. Allg. Forst. u. Jagdztg. April. 118. 1888.

Hartig R. Die pflanzliche Wurzelparasiten. Centralb. f. Bakt. u. Par.-kunde. 3:1, 19, 58, 91, 118. 1888.

-Hartig R. Lehrbuch der Anatomie und Physiologie der Pflanzen. 1891.

Hartig R. Lehrbuch der Baumkrankheiten. 2Ed. 1889.

Hartig Th. Jahresberichte über die Fortschritte der Forstwissenschaft und forstliche Naturkunde. 1837.

Hartig Th. Vollstahdige Naturgeschichte der Forstlichen Kultur-Pflanzen. 365. 1851.

Hartig Th. Luft-Boden und Pflanzenkunde in ihrer Anwendung auf Forstwissenschaft (und Gartenbau). Lehr-

- buch der Forster. 8th Ed. 1:1840. 9th Ed. 1851. 11th Ed. 1877.

Hartig Th. Ueber das sogenannten Absterben der Haarwurzeln (racines chevelues Duham.) Bot. Ztg. 21:289. 1863.

Hartig Th. Anatomie und Physiologie der Holzpflanzen. 1878.

Harting P. und Gunning. Onderzoekingen over de bron der Stickstoff voor de Planten en het ammoniak gehalte der damp-kringslucht. Amsterdam Verslag. Akad. 3:38. 1855 .

Harting P. Tegenbedenkingen op het betoog von der Herr G. J. Mulder van Waar pekomen de niet bemesten Planten hare Stickstoff? Amsterdam Verslag. Akad. 3: 89.1855.

Harting P. Recherches concernant l'assimilation de l'azote de l'air par les végétaux. Compt. rend. 41:924. 1855.

$\checkmark$ Harz C. 0. Ueber vergleichende Stickstoffdüngungsversuche. Bot. Centralb. 33:218. 1888. 
Heiden E. Ueber das Absorptions-Vermögen des Bodens und die Aufnahme der Pflanzen Nahrstoffe in der Pflanze. Ann. Landw. 41:111, 227, 343. 1863.

Hellriegel and Wilfarth. Absorption of nitrogen by plants. Bied. Centralb. 228. 1888.

Hellriegel. Welche Stickstoff Quellen stehen den Pflanzen zu Gebote? Tageblatt d. Naturforscher Versamml. z. Berlin 290. 1886.

Hellriegel und Wilfarth. Untersuchungen uber die Stickstoffnahrung der Gramineen und Leguminoseen. 1888. Berlin.

Hellriegel. Ueber die Beziehungen der Bakterien zu der Stickstoffernährung der Leguminosen. Zeitschr. f. d. Ver. f. Rübenzucker Industrie d. deut. Reiches. 241. 1886. Also, Nov. 1888.

Hellriegel. Ueber die Stickstoffnährung landwirtschäftliche Cultur gewächse. Bericht. Wien. 1890.

Hellriegel und Wilfarth. Erfolgt die Assimilation des freien Stickstoffs durch die Leguminosen unter Mitwirkung niederer Organismen? Ber. d. deut. Bot. Ges. 7:138. 1889.

Hellriegel H. Bemerkungen zu dem Aufsatze von B. Frank "Ueber den Einfluss welchen das Sterilisiren der Erdbodens auf die Pflanzenentwickelung ausübt." Ber. d. deut. Bot. Ges. 7:131. 1889.

Henschel G. Ist die zu Mycorhizabildungen führende Symbiose an jungen Fichtenpflanzen schädlich? Oest. Vierteljahrschr. f. Forstwesen. 113. 1887.

Hiltner. See Nobbe, Schmid and Hotter.

Hoffman H. Ueber Bacterien. Bot. Ztg. 27:265. 1869.

Hofmeister W. Allgemeine Morphologie der Gewachse. 1868.

Hotter. See Nobbe, Schmid and Hiltner.

Houzeau A. Faits pour servir a l'histoire de la nitrification. Compt. rend. 68:821. 1869.

Houzeau A. Faits pour servir a l'histoire de la nitrification. Ann. d. Chim. 25:161. 1872.

$\checkmark$ Immendorf. Beitrage zur Lösung der Stickstoff frage. Landw. Jahrb. 21: Hft. 1 and 2. 1892.

Irmisch. Beitrage zur Biologie und Morphologie der Orchideen. Leipzig. 1853.

Jackson C. T. Existence of nitrogen in plants; its origin in animals. Boston Med. and Sc. Jour. 63:289. 1860. 
Jager G. Ueber eine krankhafte Veränderung der Blüthen Organe der Weintraube. Flora 43:49. 1860.

Janczewski E. V. Zur parasitische Lebensweise des Nostoc lichenoides. Bot. Ztg. 3():73. '1872.

Janczewski E. V. Das Spitzenwachstum der Phanerogamen wurzeln. Bot. Ztg. Vorl. Mitth. 32:113. 1874.

Jodin F. V. Du role physiologique de l'azote. Compt. rend. 55: 912.1892.

Johow F. Die Chlorophyllfreien Humusbewohner West Indiens, biologisch-morphologisch dargestellt. Pringsheim's Jahrb. f. Wiss. Bot. 16: 445.1885.

Johow F. Die chlorophyllfreien Humus pflanzen nachi hren biologischen und anatomischen entwickelungsge schichten Verhältnissen. Pringsheim's Jahrb. f. Wiss. Bot. 20: 475. 1889.

Jönnson. Studier öfver algaparasitism hos Gunnera L. Bot. Notiser. Hft. 1:1 1894.

Joergensen A. Bidrag til Rodens Naturhistorie. Bot. Tidsskr. 10: 144.1878.

Joergensen A. Korkdanelsen paa Roden. Bot. Tidsskr. 11: 135.1879.

Jumelle H. Recherches physiologiques sur les Lichens. Rev. Gen. d. Bot, 4:49, 103, 159, 220, 259, 320. 1892.

Kámienski Fr. Die Vegetationsorgane der Monotropa hypopitys. Bot. Ztg. 39:457. 1881.

Kamienski Fr. Les organs vegetatifs du Monotropa hypopitys Mem. d. l. Soc. Nat. d. Sc. Nat. d. Cherbourg. 24: 1882.

Kamienski Fr. Ueber symbiotische Vereinigung von Pilzmycelium mit den Wurzeln höherer Pflanzen. Arbeit. d. St. Petersb. Naturf. Ges. 17:34. 1886.

Keller. Die Wurzelknöllchen der Leguminosen. Biol. Centralb. 9:97. 1889.

Kellner 0. Ueber den Gehalt einiger Wurzelgewächse an Stickstoffhaltigen Nicht-Proteinstoffen. Deut. Landw. Presse No. 82.1880.

Kellner und Yoshii. Ueber die Entbindung freien Stickstoffs bei der Faulniss und Nitrification. Zeitschr. f. Phys. Chem. 12:95. 1888.

Kerner von Marilaun A. Schmarotzer, etc. Pflanzenleben 1: $52,59,147,224,553,556,695,711,728,729,730.1890$ Also 2:77, 83, 445.1891. 
Mac Dougal: FIXATION OF FREE NITROGEN.

Kionka H. Die Wurzelknöllchen der Leguminosen. Biol. Centralb. 11: 282.1891.

Klebahn H. Die neuesten Untersuchungen uber die Wurzelknöllchen. Humboldt 148. 1890.

Klein und Szabo. Zur Kenntniss der Wurzeln von Aesculus hippocastaneum L. Flora 63:147, 163. 1880.

Kny L. Ueber parasitische Algen. Bot. Ztg. 31:139. 1873.

Kny L. Ueber die Wurzelanschwellungen der Leguminosen und ihre Erzeugung durch Einfluss von Parasiten. Sitzungsber. d. bot. Ver. d. Prov. Brandenburg 21:55. 1879.

Kny L. Zu dem Aufsatze des Herrn Prof. B. Frank "Ueber Parasiten in den Wurzelnanschwellungen der Papilionaceen." Bot. Ztg. 37: 537. 1879.

Knny L. Ueber eigenthümlich Durchwachsungen an den Wurzelhaaren zweier Marchantiaceen, untersucht von Dr. Bottger. Sitzungsber. d. Prov. Brand. Jan. 1879.

Koch und Mayer. Ueber die Aufnahme von Ammoniak durch oberirdische Pflanzentheile. Bied. Centralb. f. Landw. Sept. Hft. 1873.

Koch A. Zur Kenntniss der Fäden in den Wurzelknöllchen der Leguminoseen. Ber. d. deut. Bot. Ges. 5:111. 1887.

Koch A. Zur Kenntniss der Fäden in den Wurzelknöllchen der Leguminoseen. Bot. Ztg. 48:597. 1890.

Koch und Kossowitsch. Ueber die assimilation von freien Stickstoff durch Algen. Bot. Ztg. 51:321. 1893.

\section{Koenig. Stickstoff Vorrat. 1887.}

Kolaczek. Lehrbuch der Botanik. 3741. 1856.

Kossowicz. Die Herkunft des Stickstoffs in den Pflanzen. Mitth. Landw. Akad. zu Petrowsky 13:61. 1890.

Kossowitsch P. Durch welche Organe nehmen die Leguminosen den freien Sticksotff auf. Bot. Ztg. 50:697, 713, 729, 745, 771. 1892.

Kossowitsch P. Untersuchungen üeber die Frage ob die Algen freien Stickstoff fixiren. Bot. Ztg. 52:97. 1894.

Kostycheff. "The formation and properties of vegetable mould." St. Petersburg Soc. Nat. Bot. 20:123. 1889.

LKuhn V. R. Untersuchungen über die Anatomie der Marattia aceen und andere Gefäss-Kryptogamen. Flora 72:457. 1889.

Lachmann J. Ueber Knöllchen der Leguminosen. Landw Mittheil. Zeitschr. d. K. Lehranstalt und Versucht sta 37. 1856. 
Lachmann J. Knollen an den Wurzeln der Leguminosen. Chem. Centralb. No. 18.1892.

Laurent E. Sur le microbe des nodosités des Leguminéuses. Compt. rend. 111:750. 1890.

Laurent E. Experiences sur la production des nodosités chez. le pois a la suite d'inoculations. Bull. d. 1. Acad. Roy. d. Belgique 3 Ser. 1: No. 6. 764. 1890.

Laurent E. Expériences sur la réduction des nitrates par les végétaux. Ann. Inst. Past. No. 7:22. 1890.

Laurent E. Recherches sur les nodosites radicles. Ann. Inst. Past. 5:105. 1891.

Laurent E. Experiences sur la reduction des nitrates par les vegetaux. Rev. Mycol. 16:25. 1894.

Laurent. See Schloesing.

Lawes and Gilbert. On the present position of the question of the sources of nitrogen in vegetation, with some new results, and preliminary notice of new lines of investigation. Proc. Roy. Soc. 42: No. 256. 1886.

Lawes, Gilbert and Pugh. Assimilation of nitrogen by plants. Rep. Brit A. A. S. 51.1852.

Lawes and Gilbert. On the sources of the nitrogen of vegetation, with special reference to the question whether plants assimilate free or uncombined nitrogen. Proc. Roy. Soc. 10: 544 . 1859-60.

Lawes and Gilbert. On the sources of the nitrogen of vegetation, with special reference to the question whether plants assimilate free or uncombined nitrogen. Phil. Trans. 431. 1861. Chem. Soc. Journ. 1:100. 1863.

Lawes and Gilbert. On assimilation of nitrogen. Chem. News, 6:127. 1863.

Lawes and Gilbert. Assimilation of nitrogen by plants. Journ. d. Pharm. 44:281. 1863.

Lawes and Gilbert. Sources of nitrogen in vegetation. Proc. Roy. Soc. 43:108. 1887.

Lawes and Gilbert. On the present question of the sources of nitrogen in vegetation, etc. Phil. Trans. Roy. Soc. London, 180:1-107. 1888.

Lawes and Gilbert. Fixation of free nitrogen. Proc. Roy. Soc. 47: No. 1, 85. 1890.

Lawes and Gilbert. New experiments on the question of the fixation of free nitrogen. Proc. Roy. Soc. Lond. 47: 85. 1890 . 
Mac Dougal: FIXATION OF FREE NITROGEN.

Lawes and Gilbert. Experiments on root tubercles and the fixation of atmospheric nitrogen. Abth. f. Ag. Chem. u. Landw. Ver. d. Ges. deut. Naturf. u. Aerzte. Halle. Sept. 1891.

Lawes and Gilbert. Sources of nitrogen in leguminous plants. Jour. Rev. Ag. Soc. 3 Ser. 2:657. 1892.

LeComte H. Note sur le Mycorhiza, Bull. d. Soc. Bot. d. France 34:30. 1887.

Leone und Magnanimi. Nitrification des organischen Stickstoffs. Chem. Centralb. 1: No. 5. 1891.

Leone T. Nitrificazione e denitrificazione nella terra végétale. Gaz. Chim. ital. 20:149. 1890.

Leplay H. Sur la formation des acides organiques, des matieres organiques, azotées, et du nitrate de potasse, etc. Compt. rend. 106: 1020. 1888.

Levy J. Beitrag zur Lehre von d. Stickstoffaufnahme der Pflanzen, Dissertation. 1889. Halle.

Lehman C. Origin of nitrogen in plants. Physiol. Chem. 3: 184. 1854.

Liebig J. von. Die organische Chemie in ihrer Anwendung auf Agricultur und Physiologie. 4:164. 1842. Braunschweig.

-Liebig J. von. Die Pflanze, Liebig Annalen 121:165. 1862. Liebig J. von. Ueber die Stickstoff-haltigen Nährungsmittel des Pflanzenreichs. Liebig Annalen 39:129. 1841.

LLiebig J. von. Ueber die Stickstoff-haltigen Nährungsmittel des Pflanzenreichs Ann. d. Chem. 4:186. 1842.

Kiebig J. von. Ueber die Stickstoff-haltigen Nährungsmittel des Pflanzenreichs. Sturgeon Ann. Electr. 10:483. 1843.

Liebig J. von. Ueber die Stickstoff-haltigen Nahrungsmittel des Pflanzenreichs. Taylor Sc. Mem. 3:244. 1843.

Liebscher. "Assimilation of nitrogen by leguminous and nonleguminous plants." Deut. landw. Presse No. 104. 1080. 1892.

Liebscher. " A contribution to the nitrogen question." Deut. landw. Presse 419. 1893, and Jour. Landw. 41:139. 1893.

Liebscher. Nochmals die Stickstoff-frage. Deut. Landw. Presse. 20:1037. 1893.

Link H. F. Grundlehre der Anatomie und Physiologie der Pflanzen. 1807.

- Link H. F. Grundlehre der Krauterkunde. 1837. 
Loew 0. Ueber die Ernährungsweise des nitrificirenden Spaltpilzes Nitromonas. Bot. Centralb. 46:222. 1891.

Loew 0. "Nitrification by a Schizomycete." Bot. Ver. Munchen. April 20. 1891.

.Loew 0.' The synthetical powers of micro-organisms, Science 23: 144.1894.

Lonay A. La question de l'azote et culture les Léguminéuses, 1889. Nivelles.

Lotsy J. P. A contribution to the investigation of the assimilation of free atmospheric nitrogen by White and Black Mustard. Dept. Ag. Bull. 18. 1894.

Lotsy J. P. On the assimilation of free nitrogen by plants. Johns Hopkins Univ. Cir. 13: No. 101, 30. 1894.

Lundstroem A. N. Om mycodomatier pa papilionaoernads rötter. Bot. Notiser No. 5. 1887.

Lundstroem A. N. Ueber symbiotische Bildungen bei den Pflanzen. Bot. Centralb. 28:282. 1886.

Lundstroem A. N. Ueber Mycodomatien in den Wurzeln der Papilionaceen. Bot. Centralb. 33:159, 187. 1888.

Also Naturv. Studentsällsk. April 28, 1887. Upsala.

Lundstroem A. N. Vort. Pflanzen biologische Studien 2:71.

Lundstroem A. N. Einige Beobdachtungen ueber Calypso borealis. Bot. Centralb. 38:697. 1889.

MacDougal D. T. Nitrogen assimilation by Isopyrum biternatum. Minn. Bot. Stud. Pt. 2. 39. 1894.

Magnanimi, Leon und, Nitrification des organischen Stickstoffs. Chem. Centralb. 2: No. 5. 1891.

Magnus P, Nostoc lichenoides nicht parasitic. Bot. Ztg. 31: 251. 1873.

Magnus P. Durch Parasiten veranlasste Wurzelauswachse. Sitzber. d. Bot. Ver. d. Prov. Brand. 79. 1878.

Magnus P, Ueber Schinzia cypericola in d. Wurzeln von Cyperus flavescens und Juncus bufonius. Verhandl. d. Bot. Ver. d. Prov. Brand 21. 1879.

Magnus P. Drei neue Pilze. Ver. d. bot. Ver. f. d. Prov. Brand. 20:5C. 1878. Also 22:119. 1880.

-Magnus P. Wurzelanschwellungen des Rubus Idaeus. Ber. a. d. Hauptversamml. d. Bot. Ver. d. Prov. Brand. z. Berlin October 29, 1881.

Magnus P. Ueber einige Arten der Gatt. Schinzia Ber d. deut. Bot. Ges. 6:100. 1888. 
Magnus P. Das Auftreten der Schinzia cypericola P. Magn. in Bayern und einiges über deren Verbreitung in Europa. Separat. 1893. Berlin.

Malphigi. Anatomie plantarum pars sec. de Gallis. Op. 2:126. 1687. Leiden.

Marcano et Muntz. Sur la formation des terres nitreés dans les regions tropicales. Jour. d. Pharm. et. Chem. 5 Ser. 12: No. 61885.

Marchal E. Sur la production de l'ammoniaque dans le sol par les microbes; also Ann. d. l. Soc. Belg. d. Mic. 18: 69. 1893.

Marchand L. Sur une Nostochineé parasiteé. Bull. Soc. bot. d. France 26:336. 1879.

Masse G. On the structure and functions of the subterranean parts of Lathraea squamaria. Jour. Bot. 24: 257. 1887.

Mattei. Ancora sull' origine della Vicia faba. 1887. Bologna.

Mattirolo 0. Sul parassitismo dei Tartuffe sulla quistione delle Mycorhizae: Malphigia 1: Fasc. 8, 9. 1887.

Mattirolo 0 . Si contengono bacteri nei tubercoli radicali delle Leguminose. Malphigia 1:Fasc. 10. 464 and 11, 536. 1887.

Mattirolo 0. Sur le parasitisme des truffes et sur la question des Mycorhiza. Arch. Ital. Biol. 9:218. 1888.

Mattirolo 0. Sur le parasitisme des truffes et sur la question des mycorhizes. Arch. Ital. d. Biol. 9. 1888.

- Mayer A. Lehrbuch der Agriculturchem. 3rd Ed. 1:163. -McMurtrie. Notes on absorption of nitrogenous nutriment by the roots of plants. Proc. Brit. A. A. S. 139. 1887.

Mene C. Experiences sur l'influence d. gaz dans la végétation, Dingler polytech. Jour. 119:453. 1851.

- Mene C. Experiences sur l'influence du gaz azote dans la végétation. Compt. rend. 32:180 1891.

-Menozzi. See Alpe.

Merker P. Gunnera macrophylla Blum. Inaug. Diss. Marburg, 1888. Flora 72:211. 1889.

- Meyen J. Ueber das Hervorwachsen parasitischer Gebilde aus den Wurzeln anderer Pflanzen. Flora 12:49. 1829.

Meyer A. Ueber die Knollen der einheimischen Orchideen. Archiv. d. Pharmacie 24. 1886.

IIilde. Notizen zur Kenntniss von Anthoceros und Blasia. Bot. Ztg. 9:629. 1851. 
-Miles. Die nitrifizirenden Mikroben, Chem. Centralb. No 47. 1887.

Miles. Biological factors in the nutrition of farm crops. Proc. A. A. A. S. 433.1890.

Millon E. Nitrification. Chem. News 2:No. 56. 263, 337. 1860. See also Compt. rend. 17:452, 470 .

Millon E. Theorie chimique de la nitrification. Jour. d. Pharm. 38:325. 1860.

Millon E. Note sur la nitrification, etc. Compt. rend. 51:819. 1860.

4 Millon E. De la nitrification en la Algerie. Jour. de Pharm. 38: 246.1860.

Millon E. Theorie chimique de la nitrification. Compt. rend. 51: 548.1860.

Millon E. Theorie chimique de la nitrification. Chem. News 2:337. 1860.

Millon E. Nitrification. Chem. News. 82. 1864.

Moebius. Ueber endophytic Algen. Biol. Centralb. 11: 546. 1891.

Moebius. Conspectus algarum endophyticum. La Notarisia 6: 1221, 1279, 1291. 1891.

Moeller H. Plasmodiophora Alni. Ber. d. deut. Bot. Ges. 3: 102. 1885.

Moeller H. Beitrag zur Kenntniss der Frankia subtilis. Brunch. Ber. d. deut. Bot. Ges. 8:215. 1890.

Moeller H. Bemerkungen zu Frank's Mittheilung urber den Dimorphismus der Wurzelknöllchen der Erbse. Ber. d. deut. Bot. Ges. 10:242. 1892.

Moeller A. Ueber die Cultur flechtenbildener Ascomyceten ohne Algen. Inaug. Diss. Münster. 1887.

Horck. Ueber die Formen der Bakterioiden bei der einzelnen spezies der Leguminosen. Inaug. Dissert. 1891. Leipzig.

Morren C. Experiences sur l'absorption de l'azote par les animalcules et les algues. Compt. rend. 38:932. 1854.

Morren C. Experiences sur l'absorption de l'azote par les animalcules et les algues. Ann. Soc. Nat. (Zool.) 1:339. 1854.

Mulder G. H. Ueber die condensation des Stickstoffs aus der Atmosphäre in der Ackererde und ueber das Ernährungsvermögen der organische Bestandtheile des Bodens für die Pflanze. Jour. f. Prakt. Chem. 32: 344. 1844. 
Mulder G. H. Ueber die Quelle des Sticks toffs in den Pflanzen. Flora 28:239. 1845 .

- Mulder G. H. Van Waar bekommen die niet bemeste planten hare Stickstoffs. Amsterdam. Verslag. 3:61. 1855.

Mulder G. H. Versuch einer Phys. Chem. 751.

Muller P. E. Bemerkungen ueber die Mycorhiza der Buche. Bot. Centralb. 26:22. 1886.

Muntz. See Schloesing.

Muntz A. De quelques faits d' oxydation et de reduction, produits par les organismes microsc. du sol. Journ. d. Pharm. et d. Chem. 5 Ser. 12:No. 6. 1885.

Muntz and Marcano. Sur la formation des terres nitreés dans les regions tropicales. Journ. d. Pharm. et d. Chem. 5 Ser. 12: No. 6.1885.

Muntz A. Ueber einige Oxidation und Reduction durch Mikroorganismen im Boden. Chem. Centralb. No. 37. 1885.

Muntz A. Ueber d. Bildung d. saltpeterhaltigen Erden in den Tropen. Chem. Centralb. No. 37. 1885.

Muntz A. Sur la decomposition des engrais organiques dans le sol. Compt. rend. 110:1206. 1890.

-Muntz A. Sur la decomposition des engrais organiques dans le sol. Compt. rend. 110: 1026.1890.

Muntz A. Du role des engrais verts comme furnures azoteés. Compt. rend. 110:972. 1890.

Muntz A. Sur la formation des nitrates dans la terre. Compt. rend. 112:1142. 1891.

Muntz A. Sur la formation des nitrates dans la terre. Compt. rend. 112:1143. 1891.

-Muntz and Coudon. La fermentation ammoniacale de la terre. Ann. Agron. 19: 209. 1893.

N. H. J. M. The fixation of free nitrogen. Nature, 24:41. 1890.

Naegeli. Botanische Beiträge: Pilze im Innern von Zellen Linnaea 16:278. 1842.

Naegeli. Ernährungsmechanismus der niederen Organismen. Sitzber. d. Münch. Akad. July, 1879.

Naegeli. Ernährung der niederer Pilze durch Kohlen und Stickstoffverbindungen. Bot. Mittheil. 3:395. 1881.

Nathusius. Symbiose der Waldbäume mit Mycorhiza. Zeitschr. f. Naturw. 62: Hft. 61890.

Nicolai. Das Wachstum der Wurzel. Schr. d. phys.ökön. Ges. z. Koenigsberg. 7:35, 78. '1875. 
Noack F. Ueber Micorhizende-bildende Pilze. Bot. Ztg. 47:389. 1889.

Nobbe F. Vegetationsversuche in Boden mit lokalisirten Nathrstoffen. Landw. Ver. Sta. 6:98. 1868.

LNobbe F. Ueber die Stickstoffernährung der Leguminosen. Verhandl. d. Ges. Deut. Naturf. und Aerzte. Versamml z. Bremen, 2:551. 1890.

Nobbe F. Stickstoffernährung der Leguminosen. Chem. Centralb. 1: No. 6. 1891.

Nobbe, Schmid, Hiltner and Hotter. Versuche ueber die Stickstoffassimilation der Leguminosen. Landw. Ver. Sta. 39: 327.1891

Nobbe, Schmid, Hiltner and Hotter. Ueber die physiologische Bedeutung den Wurzelknöllchen von Eleagnus angustifolius. Landw. Ver. Sta. 41:138. 1892.

LNobbe, Schmid, Hiltner and Hotter. Ueber die Verbreitungs-

1 fähigkeit der Leguminosen Bacterien im Boden. Landw. Ver. Sta. 41:137. 1892.

"Nobbe and Hiltner. "The interchange between leguminous plants and the bacteria causing root tubercles." Sächss. Landw. Zeitschr. 165. 1893.

- oliver F. W. On Sarcodes sanguinea. Ann. Bot. 4:303. 1890.

-0tto R. Die Assimalation des freien atmosphärischen Stickstoffes durch die Pflanze. Bot. Centralb. 46:387. 1890. Also, 47:62, 123, 175.1891.

0tto. See Frank.

Penzig 0. Die Krankheit der Edelkastanien und B. Frank's Mykorhiza. Ber. d. deut. Bot. Ges. 3:301. 1885.

Persoon. Traité sur les champignons comestibles. 1818.

Petermann A. "Contribution a la question de l'azote." Mem. d. l' Acad. Roy. d. Belg. 1891.

Petermann A. Beiträge zur Stickstoffrage. Abstract. Chem. Centralb. 1:988. 1893. Ibid. 2:889. 1892. Ibid. 3: No. 20.1892.

Petermann A. Contribution a la question de l'azote. Mem. d. l' Acad. roy. d. Belg. 47. 1892.

Petermann A. Contribution a la question de l'azote. Bull. Acad. Roy. Belg. 3, Ser. 25:267. 1893.

$\checkmark$ Pfeffer W. Ueber Fleischfressende Pflanzen und ueber die Ernahrung durch Aufnahme organischer Stickstoff ueberhaupt. Landw. Jahrb. 6:969. 1877.

Pfeffer W. Pflanzenphysiologie 1:239. 1880. 
${ }_{L}$ Pfeiffer A. Die Beziehungen der Boden Capillarität zum Transport von Bacterien. Zeitschr f. Hyg. 1:394. 1886.

Pfeiffer A. Antwort auf die Entgegnung des Herrn Dr. Soyka, etc. Zeitschr. f. Hyg. 2:239. 1887.

Pfeil W. Wunschenwerthe Abbildung der Baumwurzeln wie sie sich nach dem Alter des Baumes in verschiedenen Boden-andern. Krit. Bl. f. Forst. u. Jagdwiss. 25: 255. 1848.

.Pfeil W. Recension von Schacht's "Beiträge zur Anatomie und Physiologie der Gewächse." Krit. Bl. f. Forst. u. Jagdwiss. 35:51. 1855.

${ }_{C}$ Pichard P. Influence dans' les terres neues des proportions d'argile et d'azote organique sur la fixation d'azote atmospherique, sur la conservation de l'azote et sur la nitrification. Compt. rend. 114:81. 1892.

Pichi. Alcuue osservazione sui tubercoli radicali delle Leguminose. Atti della Soc. Tosc. di Sc. nat. 1888.

Pott. Untersuchungen über Wachstumisverhaltnisse der Leguminoseen. Landwirtsch. Versuchssta. 25: Hft. 1 and 2.

Poulsen V. A. Bidrag til Triuridaceernes. 1886.

Poulsen V. A. Triuris major n. sp. Et Bidrag til Triuridaceernes Naturhistorie. Bot. Tiddskr. 17:293. 1890.

-Prantl K. Untersuchungen ueber die Regeneration des Vegetationspunktes an Angiospermwurzeln. Arbeitld. bot. Inst. z. Würzburg. 1:546. 1874.

Prantl K. Die Assimilation freien Stickstoff und der Parasitismus von Nostoc. Hedw. 28:135. 1889.

Prazmowski. Ueber die Wurzelknöllchen der Leguminosen. Bot. Cent ralb. 36:215, 248, 280. 1888.

Prazmowski. Das Wesen und die biologische Bedeutung der Wurzelknöllchen der Erbse. Ber. a. d. Sitz. d. Akad. i. Krakau. 1889.

Prazmowski. Die Wurzelknöllchen der Erbse. die Aetiologie und Entwickelungsgeschichte der Knöllchen. Landw. Vers. Sta. 37:Hft. 3 and 4, 104, 161. 1890. See also 38: Hft. 5. 1890.

Prillieux E. De la structure anatomique et du mode de vegetation du Neottia nidus. avis. Ann. d. Sc. Nat. Bot. 4 Ser. 5:267. 1856.

Prillieux E. Sur la nature et la cause de la formation des tubercles qui nassent sur les racines des Leguminéuses. Bull. Soc. Bot. d. France. 26:98. 1879. 
Prillieux E. Anciennes observations sur les tubercles des racines des Leguminéuses. Compt. rend.'111:926. 1890.

$\sim$ Prillieux E. Anciennes observations sur les tubercules des racines des Leguminéuses. Bull. d. l. Soc. Bot. d. France. 13: No. 1. 1891.

Prove. Untersuchungen ueber die Stickstoffnahrung der Erbsen. Zeitschr. Landw. Ver. Bayern. 82:85. 1892.

Pugh, Lawes and Gilbert. Assimilation of nitrogen by plants. Rep. Brit. A. A. S. 51. 1852.

Rabuteau. Recherches sur les metamorphoses et l' elimination des azotites. Comp't. rend. Soc. d. Biol. 5 Ser. 1:66. 1870 .

Raulin. Etudes chimiques sur la végétation des Musedinées. Compt. rend. 57:229. 1863.

Rees M. Entstehung der Flechte-Collema glaucescens. Monatsber. d. Berl. Akad. Oct. 1871.

LRees M. Ueber den Parasitismus von Elaphomyces granulatus. Sitzungs-Ber. der Physik.-Med. Soc. z. Er. langen. May. 1880.

Rees M. Ueber Elaphomyces und sonstige Wurzelpilze. Ber. d. deut. Bot. Ges. 3:293. 1885.

Rees und Fisch. Untersuchungen ueber den Bau und Lebensgeschichte der Hirschtruffel-Elaphomyces. Biblioth. Bot. Hft. 7: 1. 1887.

Reinke J. Ueber gonidienartige Bildungen in einer dicotylischer Pflanze. Nach. v. d. konigl. Ges. d. Wiss. u. Univ. z. Göttingen, $100.1871,1872$.

Reinke J. Andeutungen ueber den bau der Wurzel von Pinus Pinea. Bot. Ztg. 30:49. 1872.

Reinke J. Morphologische Abhandlungen. 1873.

-Reinke J. Zur Kenntniss der Rhizome von Corallorhiza und Epipogon. Flora. 56:145, 161, 177. 1873.

Reinke J. Zwei parasitische Algen. Bot. Ztg. 37:473. 1879.

Reinsch H. Ueber eigerthümlichen Stoff in der Monotropa hypopitys. Jahrb. f. prakt. Pharm. u. verw. Facher, - 25:193. 1852.

Reissek S. Endophyten der Pflanzenzelle. 1846. Uren.

Resa F. Ueber die Periode der Wurzelbildung. Inaug. Diss. 1877.

Resa F. Untersuchungen ueber die Periode der Wurzelbildung, insbesondere bei den Holzgewachse. Forstl. Bl. 321. 1878. 
Reum J. A. Pflanzen-Physiologie. 1835.

Reuss H. Der Waldfeldbau im Dienste des Forstkulturbetriebs. Centralb. f. d. gesamm. Fortstw. 289, 354. 1889.

Rockwood, see Atwater.

Rossmassler. Der Wald. 418. 1863.

Rostrop E. Sygdomme hos Skovtraeerne, foraarsagede af ikkerustatige 'Snyltesvampe. Tidsskr. f. Skovbrog. 4:113. 1880 .

Russel H. L. The fixation of free nitrogen by plants Bot. Gazette. 19:284. 1894.

-Russow. Vergleichende Untersuchungen der Leitbündelkryptogamen. 122. 1872. St. Petersburg.

Rylands T. G. On the nature of the byssoid substance found investing the roots of Monotropa Hypopitys. The Phytologist $1: 341$. 1842.

¿Sarauw F. L. Ueber die Mykorhizen unserer Waldbaume. Bot. Centralb. 53:343. 1893.

Larauw F. L. Rödsymbiose og mykorhizer saerlig hos skovtraerne. 1893.

-Sachs J. Zur Entwickelungsgeschichte d. Collema bulbosa. Bot. Ztg. 13:1. 1855.

-Sachsse. Keimung von Pisum sativum. 1872.

Serno. Ueber das Auftreten und des Verhalten der Salpetersaure in den Pflanze. Landw. Jahrb. 18:877. 1889.

-Schacht H. Physiologische Botanik. die Pflanzenzelle. 1852.

Schacht H. Beitrag zur Entwickelungsgeschichte der Wurzel. Flora 36:261. 1853.

Schacht H. Ueber Pilzfäden im innern der Zellen und der Starkemehlkörner. Ber. u. d. Verh. d. königl. preuss. Akad. d. Wiss. z. Berlin. 377. 1854.

Schacht H. Beitrag zur Anatomie und Physiologie der GeWächse. 160. 1854.

Schacht H. Lehrbuch d. Anatomie und Physiologie. 2:147. 1859.

Schacht H. Der Baum 172. 1860.

Schenk. Symbiose einer Ericaceae und Mycorhiza. Zeitschr. f. Naturw. 62:Hft. 6.1890.

-Schenk. Ueber Cycas Wurzeln mit Algencolonien. Tagebl. d. 45 Versamml. deut. Naturf. u. Aerzte z. Leipzig. 1872.

Schindler. Ueber die biologische Bedeutung der Wurzelknöllchen bei der Papilionaceen. Jour. f. Landw. 33:325. 1885 . 
Schindler. Ueber die Bedeutung die sogenannten Wurzelknöllchen bei der Papilionaceen. Oester. Landw. - Wochenb. 11: No. 34. 1885.

$\checkmark$ Schindler. Zur Kenntniss der Wurzelknöllchen der Papilionaceen. Bot. Centralb. 18:86. 1888.

Schlechtendal. Phaseolus multiflorus. Bot. Ztg. 10:893. 1852.

Schleiden M. J. Grundzüge der wissenschaftliche Botanik. 1842-43. 1845-46. 1849-50.

Schlicht A. Ueber neue Fälle von Symbiose der Pflanzenwürzeln mit Pilzen. Ber. d. deut. Bot Ges. 269. 1888.

Schlicht A. Beitrag zur Kenntniss der Verbreitung und der Bedeutung der Mykorhizen. Inaug. Diss. Univ. Erlangen. 1889.

Schloesing and Iuntz. Sur la nitrification par les ferments organises. Compt. rend. 80:1250, 1873; 84:301, 1877; 85:1018, 1878, and 86:891, 1879 .

Schloesing Th. Sur les relations de l'azote atmosphérique avec la terre végétale. Compt. rend. 106:805, 898, 982, 1123. 1888; also 10\%:290. 1888.

Schloesing Th. Sur la nitrification de l'ammoniaque. Compt. rend. 109:423, 883. 1889.

Schloesing Th. Sur les relations de l'azote atmosphérique avec la terre végétale. Compt. rend. 109:210, 345. 1889.

Schloesing and Laurent. Sur la fixation de l'azote gazeux par les Legumineuses. Compt. rend. 111:750, 1800; also 115 : 659, 732, 1892.

-Schloesing Th. Remarques au sujet des observations de M. Berthelot, etc. Compt. rend. 110:612. 1890.

Schloesing Th. Sur l'absorption l'ammoniaque de l'atmosphere par la terre végétale. Compt. rend. 110:429, 499.1890.

Schloesing and Laurent. Sur la fixation de l'azote libre par les plantes. Compt. rend. 113:776. 1891.

Schloesing Th. Observations sur la communication de $\mathbf{M}$. Berthelot. Compt. rend. 115:636. 1892.

Schloesing and Laurent. Recherches sur la fixation de l'azote libre par les plantes. Ann. Inst. Past. 6: 65. 1892.

Schmid. See Nobbe, Hiltner and Hotter.

Schmitter. "New experirnents in soil inoculation." Wochenschr. ökön. Ges. 251. 1891. 
Schmitz Fr. Phyllosiphon Arisari. Bot. Ztg. 40:523, 539, 566, 579. 1882.

Schneider A. Observations on some American Rhizobia. Bull. Torr. Bot. Club 19:205. 1892.

-Schneider A. Recent investigations concerning rhizobia and free nitrogen assimilation. Ag. Science 7:549. 1893.

Schneider A. A new factor in Agriculture. University of Illinois. Ag. Ex. Sta. Bull. No. 29, December, 273. 1893.

Schneider A. Morphology of the root tubercles of the Leguminosae. Am. Naturalist 27:782. 1893.

Schneider A. Beitrag zur Kenntniss der Rhizobien. Ber. d. deut. Bot. Ges. 12:11. 1894.

Solms-Laubach. Der Aufbau des Stockes von Psilotum triquetrum und dessen Entwickelung aus der Brutknospe. Ann. d. Jard. Bot. d. Buitenzorg. 4:139. 1884.

Sorauer P. Wurzelknollen. Pflanzenkrankheiten 1:743. 1886.

Vorauer P. Die Hernie der Erlenwurzeln. Pflanzenkrankheiten 2:73. 1886.

Sorauer P. Zusammenstellung der neuern Arbeiten ueber die Wurzelknöllchen und deren als Bakterien ausgesprochene Inhaltskörperchen. Bot. Centralb. 31:308, 343, 1887.

Schuetzler J. B. Sur les germes organises de la nitrification. Bull. Soc. vand. d. Sc. Nat. Lausanne. 22:213. 1886.

Schulze E. Ueber die Processe durch welche in der Natur ein freier Stickstoff in Stickstoffverbindungen übergegeführt wird. Landw. Jahrb. 6: Hft. 4 and 5. 1877.

Schwarz F. Die Wurzelhaare der Pflanzen. Unters. a. b. bot. Inst. z. Tub. 1 : Hft. 1, 135.1883.

Schwendener S. Ueber die wahre Natur der Flechten. Verh. d. schweiz. naturf, Ges. z. Rheinfelden. Sept. 1867.

Schwendener S. Untersuchungen ueber den Flechtenthallus. Nachtrag Nageli, Beitrag zur wiss. Bot. 4:195. 1868. -Schwendener S. Die Algentypen der Flechtengonidien. 1869. -Soyka J. Der Boden. Handbuch d. Hygiene u. d. Gewerbkrankheiten. 1: Abt. 2 Hft. 3. 1887.

-Soyka J. Entgegnung auf Herrn Dr. A. 'Pfleiffer's Aufsatz "Die Beziehungen der Boden Capillaritat." Zeitschr. f. Hyg. 2:96. 1887.

Storer. Agriculture, 1:33,309, 420, 467, etc. 1892 . Boston.

Tacke B. Ueber die Entwickelung von Stickstoff bei Faulniss. Landw. Jahrb. 16:917. 1887. 
Tacke B. Ueber den Stickstoffverlust bei der Nitrification und den Stickstoffgewinn im vegetations freien Erdboden. Landw. Jahrb. 18:439. 1889.

Thomai K. Bildung der Eiweisskörper in der Pflanze. Naturw. Wochenschr. 8:469. 1892.

Thomas M. B. The Genus Corallorhiza. Bot. Gazette 18:166. 1892.

Treviranus L. C. Ueber die Neigung der Hülsengewächse zu unterirdischen Knollenbildung. Bot. Ztg, 11:393. 1853.

Thumen von. Les Mycorhizes et les théoriés nouvelles de la vie complexe biologie. Rev. gen. d. Sc. Pures et Appl. 1: 326 .

Tieghem P. van. Recherches sur la symmetrie de la structure des plantes vasculaires. Ann. d. Sc. Nat. Bot. 5 Ser. 13: 5.1871.

Tieghem P. van. Sur le reseau de soutien de l'ecorce de la racine. Ann. d. Sc. Nat. Bot. 7 Ser. 7:375. 1888.

Tieghem van and Douliot. Recherches comparatives sur l'origine des membres endogenes dans les plantes vascuculaires. Ann. d. Sc. Nat. 7 Ser. 8:1. 1888.

Treub M. Korte bot. aanteekenimgen II; Nostoccolonien in Gunnera macrophylla Blum. Nederland. Kruidkrund. Archief. 2 Ser. 3:407. 1882.

-Treub M. Etudes sur les Lycopodiaceés. Ann. d. Jard. d. Bot. Buitenzorg 5:87. 1886.

Troschke. Ueber der Kultur der Lupin im wasserige Nahrlosung und ueber der Wurzelanschwellungen der Lupine. Mittheil a. d. Vers. Sta. Regenw. 1884.

Troschke. Die gegenwärtigen stand der Stickstoffrage. Wochenschr. d. pömmerschen ökon. Ges. 24:4. 1884.

Tschirch. Beiträge zur Kenntniss der Wurzelknöllchen der Leguminosen. Ber. d Bot. Ges. 5:58. 1887.

Tschirch. Ueber der Wurzelknöllchen der Leguminosen. Ges. Naturw. Freunde z. Berlin No. 4. 1887.

Tschirch. Ueber den Einfluss des Sterilisirens des Erbodens auf die darin wächsenden Pflanzen. Tagebl. d. 60 Versamml. deut. Naturf. u. Aerzte i. Wiesbaden, Sept. 1887.

Tschirch. Ueber die Untersuchungen des Herrn Frank: Ueber die Wurzelsymbiose der Ericaceen. Tagebl. d. 60 Versamml. deut. Naturf. u. Aerzte i. Wiesbaden, Sept. 1887. 
Tschirch. Ueber die Entwickelungsgeschichte der Bakterioiden in der Wurzeln der Leguminosen. Tagebl. d. 60 Versamml. deut. Naturf. u. Aerzte i. Wiesbaden, Sept. 1887.

Tubeuf C. V. Beiträge zur Kenntniss der Baumkrankheiten. 1888. Berlin.

Tulasne L. Observations sur le genre Elaphomyces. Ann. d. Sc. Nat. Bot. 2 Ser. 16:5. 1841.

Tulasne L. Fungi Hypogaei. 1857.

-Unger F. Beiträge zur Kenntniss der parasitischer Pflanzen. Ann. e. Wien. Mus. d. Naturgesch. 2:13. 1840.

Unger F. Versuche ueber die Ernährung der Pflanzen. Flora $25: 2,241.1842$.

Unger F. Ursprung des von den Pflanzen ausgeschiedenen Stickstoffgas. Wien Sitzungsbar. 10:414. 1853.

Unger F. Die Pflanze und die Luft. Skofitz. Bot. Wochenbl. 3: 187.1854.

Vannuccini G. Sull' assorbimento e diffusione dell' azoto e dei nitrati nel terreno privo di vegetazione. Anghiari. 1891.

Ville G. Note sur l'assimilation de l'azote de l'air par les plantes. Compt. rend. 31:578. 1851.

-Ville G. Note sur l'assimilation de l'azote de l'air par les plantes. Jour. f. Prakt. Chem. 52:60. 1851.

Ville G. Recherches experimentales sur la végétation. Comp̀t. rend. 35:464, 650. 1852.

Ville G. Recherches experimentales sur la végétation. Cosmos. 1:669. 1852. Also 2:309. 1853.

Ville G. Recherches experimentales sur la végétation. Ann. Nat. Hist. 12:68. 1853.

Ville G. Recherches experimentales sur la végétation. Jour. f. Prakt. Chem. 58:10,368. 1853.

Ville G. Recherches experimentales sur la végétation. Proc. Roy. Soc. 6:307. 1853.

Ville G. Absorption de l'azote de l'air par les plantes. Compt. rend. 38: 705, 723. 1859.

-Ville G. Absorption de l'azote de l'air par les plantes. Jour. f. Prakt. Chem. 62:121, 183.1854.

-Ville G. Sur l'assimilation du gaz azote de l'air par les végétaux. Bibl. Univ. Arch. 30:305. 1855.

Ville G. Quel est le role des nitrates dans l'oconomie des plantes? Compt. rend. 41:987. 1855. Also 43:85, 612. 1856. 
Ville G. De l'etat auquelse trouve quand il est absorbe l'azote quiles plantes tirent de l'air. Compt. rend. 43:143. 1856.

VIlle G. Quel est le role des nitrates dans l'oconomie des plantes? Ann. d. Chim. et Phys. 49:168. 1857.

-Ville G. Nouvelles recherches sur le role des principes inorganiques dans l'oconomie de la nutrition végétale. Compt. rend. 47:438. 1858.

Ville G. De l'importance compareé des agents de la production végétale. Compt. rend. 51:874. 1860.

Ville G. " Nitrification." Mem. Novorossian Soc. Nat. 17:1. 1892.

Vines S. H. Note on the Nitrogenons nutrition of the Bean. Rep. Brit. A. A. S. 741.1888.

Vines S. H. On the relation between the formation of tubercles on the roots of Leguminosae and the presence of nitrogen in the soil. Ann. Bot. 2: 386. 1888-89.

Vittadini C. Monographia Lycoperdineorum. Mem. d. reale Accad. d. Sc d. Torino. 2 Ser. 5:145. 1843.

Vonhoene H. Ueber dås Hervorbrechen endogener Organe aus dem Mutterorgane. Flora. 6:227, 243, 268. 1880.

Vuillemin P. Les tubercles radicaux dés Légumineuses. Ann. d. Sc. Agron. franc. et étrang. 1:121. 1888.

Vuillemin P. Remarques sur la memoire de Lundstroem. Jour. d. Bot. April. 1888.

Vuillemin P. Antibiose et Symbiose. Ass'n. fr. pour l'avan. d. Sc. 18.1889.

$\checkmark$ uillemin P. Les tubercles des Légumineuses et leurs habitants. Bull. Soc. l. Sic. d. Nancy 2 Ser. 9: fasc. 22. 1887. Also Separate. Paris, 1889.

Vuillemin P. Les Mycorhizes. Rev. Gen. d. Sc. pures et app. 1:326. 1890.

Ward M. On the tubercular swellings on the roots of Vicia faba. Phil. Trans. Roy. Soc. 178:139. 1887.

Ward M. Some recent publications bearing on the source of nitrogen in plants. Ann. Bot. 1:325. 1887-88.

Ward M. On the tubercles of leguminous plants with special reference to the pea and bean. Proc. Roy. Soc. 46:431. 1889.

Ward M. Symbiose und Symbiotische Gährungen. Chem. Centralb. 1: No. 18.1892.

Ward M, Recent investigations and ideas on the fixation of nitrogen by plants. Nature, 49:511. 1894. 
Waage Th. Ueber haubenlose Wurzeln der Hippocastanien und Sapindaceen. Ber. d. deut. Bot. Ges. 9:139. 1891.

Wagner P. Ist es wahr das der weisse Senf den freien Stickstoff der atmosphärischen Luft aufnimmt und nach Art der Leguminosen Stickstoffbereichenden wirkt? Deut. Landw. Presse, 20:901. 1893.

LWagner P. Einige Zeit und Streitfragen aus dem Gebiet der Düngungslehre. Deut. Landw. Presse, 20:913, 933, 1004. 1893.

Wahrlich W. Beitrag zur Kenntniss der Orchideenwurzelpilze. Bot. Ztg. 44:481. 1886.

Warington. Alterations in the properties of the nitric ferments by cultivation. Chem. News, 43. 1881.

Warington. On alterations in the properties of the nitric ferment by cultivation. Rep. Brit. A. A. S. 393. 1881.

Warington. Some practical aspects of recent investigations on nitrification. Jour. Soc. Arts. Lond. 30:532. 1881-2. Also Nature, 30:644. 1884.

'Warington. On nitrates in the soil. Rep. Brit. A. A. S. 469. 1883.

W arington. On nitrification. Rep. Brit. A. A. S. 682.1884.

Warington. On nitrification. Jour. Chem. Soc. Lond. 45:537. 1884. Also Rep. Brit. A. A. S. 54:682. 1885.

Warington. On the distribution of the nitrifying organism in the soil. Chem. News. 54:228. 1886.

Warington. On the distribution of the nitrifying organism in the soil. Rep. Brit. A. A. S. 582: 1886.

-Warington. On the power of certain bacteria to form organic compounds from inorganic matter. Rep. Brit. A. A. S. 866. 1890.

Warington. Recent investigations on nitrification. Ag. Science 7: 34.1893.

Warming. Hippophaë rhamnoides. Smaa biologiske og rmorfologiske Bidrag. Bot. Tidsskrift 9:108. 1876.

- Weber C. Ueber den Pilz der Wurzelanschwellungen Juncus bufonius. Bot. Ztg. 42:369. 1884.

Wicke. Beobdachtungen an Chenopodium vulvularia über die Ausscheidung von Trimethylamin. Bot. Ztg. 20:393. 1862.

Wiesner. Biologie der Pflanzen. 1889. Wien. 
Wigand. Bacterien innerhalb der geschlossenen gewebes der knollenartigen anschwellungen der PapilionaceenWurzeln. 'Bot. Heft. Forsch. a. d. Bot. Gart. z. Marburg. Hft. 2, 88. 1887.

LWillfarth. Ueber Stickstoffaufnahme der Pflanzen. Tagebl. d. 60 Versamml. deut. Naturf. u. Aerzte i. Wiesbaden. Sept. 1887.

Willfarth. Ueber die Stickstoffaufnahme der Pflanzen. Verhandl. d. Ges. Deut. Naturf. und Aerzte. Versamml. z. Bremen. 2:549. 1890.

Willfarth. Stickstoffaufnahme der Pflanzen. Chem. Centralb. 1: No. $14 . \quad 1891$.

Willfarth. "New experiments with plants collecting nitrogen, and their employment in agricultural practice." Deut. Landw. Rundschau Nos. 8, 9, 10, 11, 12.1882. Willfarth, see Hellriegel.

Winogradsky. Sur les organismes de la nitrification. Compt. rend. 110:1013. 1890.

Winogradsky. Recherches sur les organismes de la nitrification. Ann. Inst. Pasteur No. 4, 213. 1890. No. 5, 257. 1890. No. $12,760.1890$. No. 2, 92.1891 . No. 9, 577. 1891.

Winogradsky. Sur la formation et l'oxydation des nitrites pendant la nitrification. Compt. rend. 113:89. 1891.

Winogradsky. Contributions à la morphologie des Organismes de la nitrification. Arch. d. Sc. Biol. St. Petersb. 87. 1892.

Winogradsky. Sur l'assimilation de l'azote gazeux de l'atmosphere par les microbes. Compt. rend. 116:1385. 1893.

Winogradsky. Sur l'assimilation de l'azote gazeux de l'atmosphere par les microbes. Compt. rend. 11 8:353. 1894.

Wolff und Zimmermann. Beitrag zur Chemie und Physiologie der Pilze. Bot. Ztg. 29:280. 1871.

Wollny, Ueber die Thätigkeit niederer Organismen in Boden. 1883. Braunschweig.

Wollny. Ueber die Thätigkeit niederer organismen in der Ackererde. Deut. Landw. Presse, Nos. 47, 84, 85, 87, 89. 1883. Nos. $23,24,27,28,29,31.1884$.

Wollny. Untersuchungen ueber dieZersetzung der organischen Substanzen. Jour. f. Landw. 34:213. 1886. 
Mac'Dougal: FIXATION OF FREE NITROGEN.

Wollny. Ueber die Beziehungen der Mikroorganismen zur Agrikultur. Centralb. f. Bakt. und. Par-Kunde 1: 441, 467. 1887.

-Wollny. The relation of the physical properties of the soil to the cultivation of plants. Ex. Sta. Rec. 4:528, 627. 1893.

Woods. See Atwater.

Woods C. D. The acquisition of nitrogen by growing plants. Conn. Storrs Ex. Sta. Rep. 4:17. 1891.

Woronin. Ueber die bei der Schwarzerle und der gewoehnlichen Garten-Lupine auftretenden Wurzel anschwellungen. Mem. d. l'Acad. d. St. Petersbourg, 7, Ser. 10: No. 6.1866.

Woronin. Observations sur certaines excroissances que presentant les racines d. l'aune et d. lupin des jardin. Ann. d. Sc. Nat. Bot. 5. Ser. 7:73. 1867.

Woronin. Plasmodiophora Brassicae. Pringsheim's Jahrb. $11: 548.1878$.

Woronin. Ueber d. Pilzwurzel v. B. Frank. Ber. d. deut. Bot. Ges. 3: Hft. 6.1885.

Wortman. Ueber die neuesten Untersuchungen bezüglich der organismen der Nitrifikation und ihre physiologische Bedeutung. Landw. Jahrb. 20: Hft. 1, 175. 1891.

Wydler H. Kleinere Beiträge zur Kenntniss einheimischer Gewächse: Papilionaceen. Flora 43:17, 51, 83. 1860.

-Yoshii, Kellner und. Ueber die Entbindung freien Sticktoffs bei der Faulniss und Nitrification. Ztschr. f. physiol. Chem. 12:95. 1887-88.

-Zimmermann und Wolff. Beitrag zur Chemie und Physiologie der Pilze. Bot. Ztg. 29:280. 1871. 


\section{Description of Plates.}

\section{Plate VII.}

The Anderson registering balance set up to weigh transpired water.

\section{Plate VIII.}

The Frost electric auxanometer in use to determine growth in leng th.

\section{Plate IX.}

The Frost time-recorder.

\section{Plate $\mathrm{X}$.}

The Frost auxanometer connected with recorder.

From photographs by Professor Wm. R. Appleby. 
Bulletin No. 9. MINNESOTA BOTANICAL STUDIES. Sept. 1894.

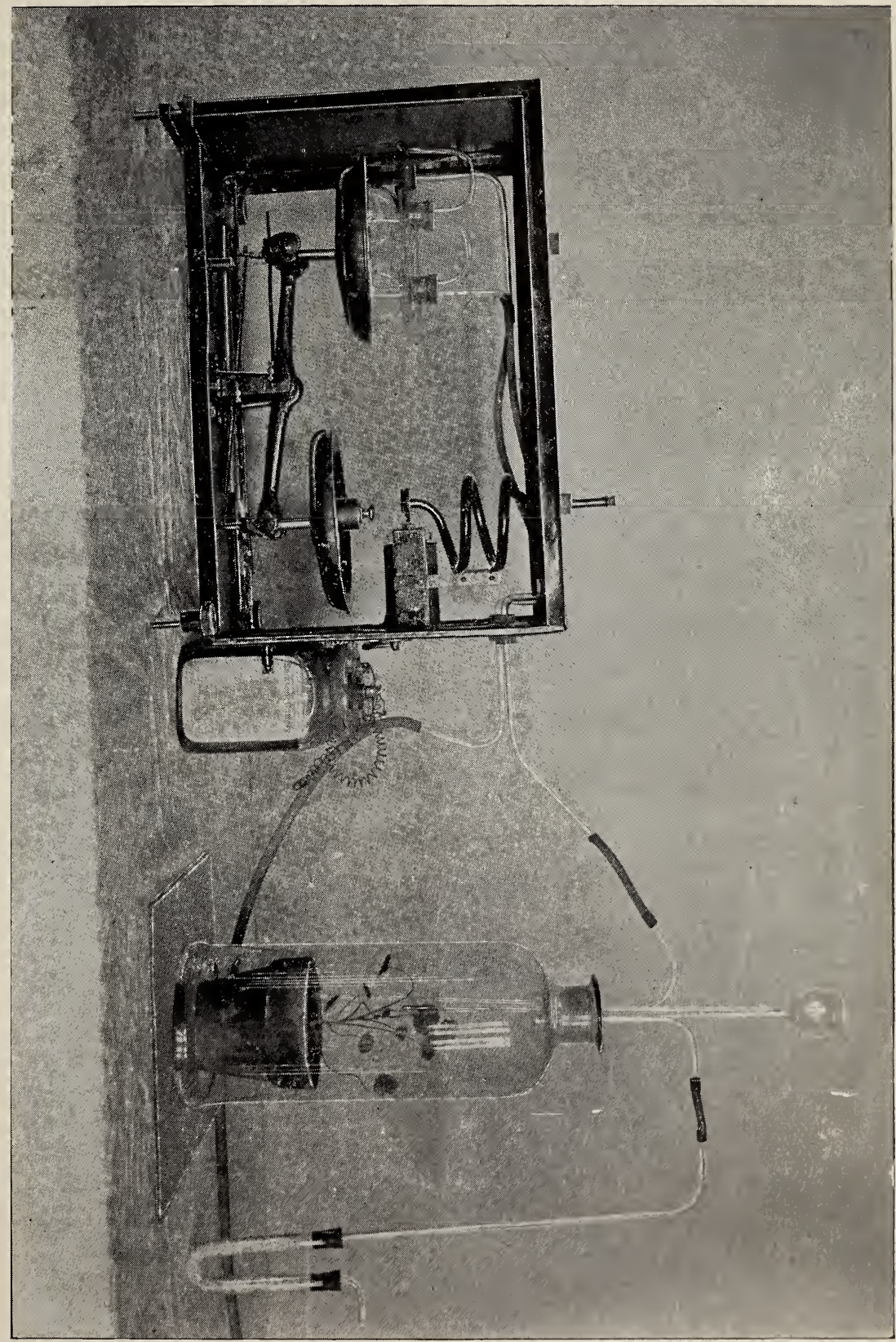

PLATE VII. 
Bulletin No. 9. MINNESOTA BOTANICAL STUDIES Sept. 1894

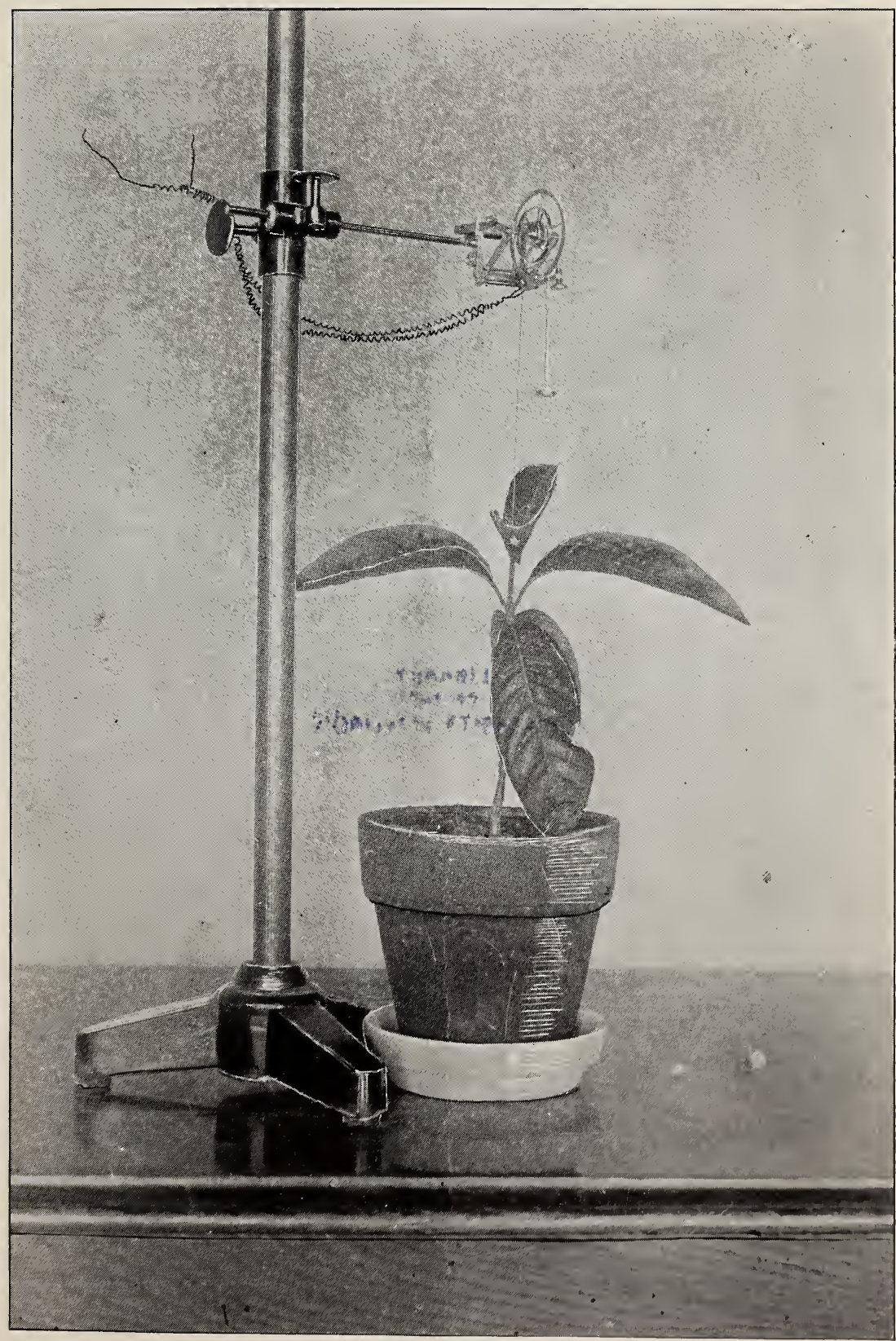

PLATE VIII. 

Bulletin No. 9. MINNESOTA BOTANICAL STUDIES. Sept. 1894.

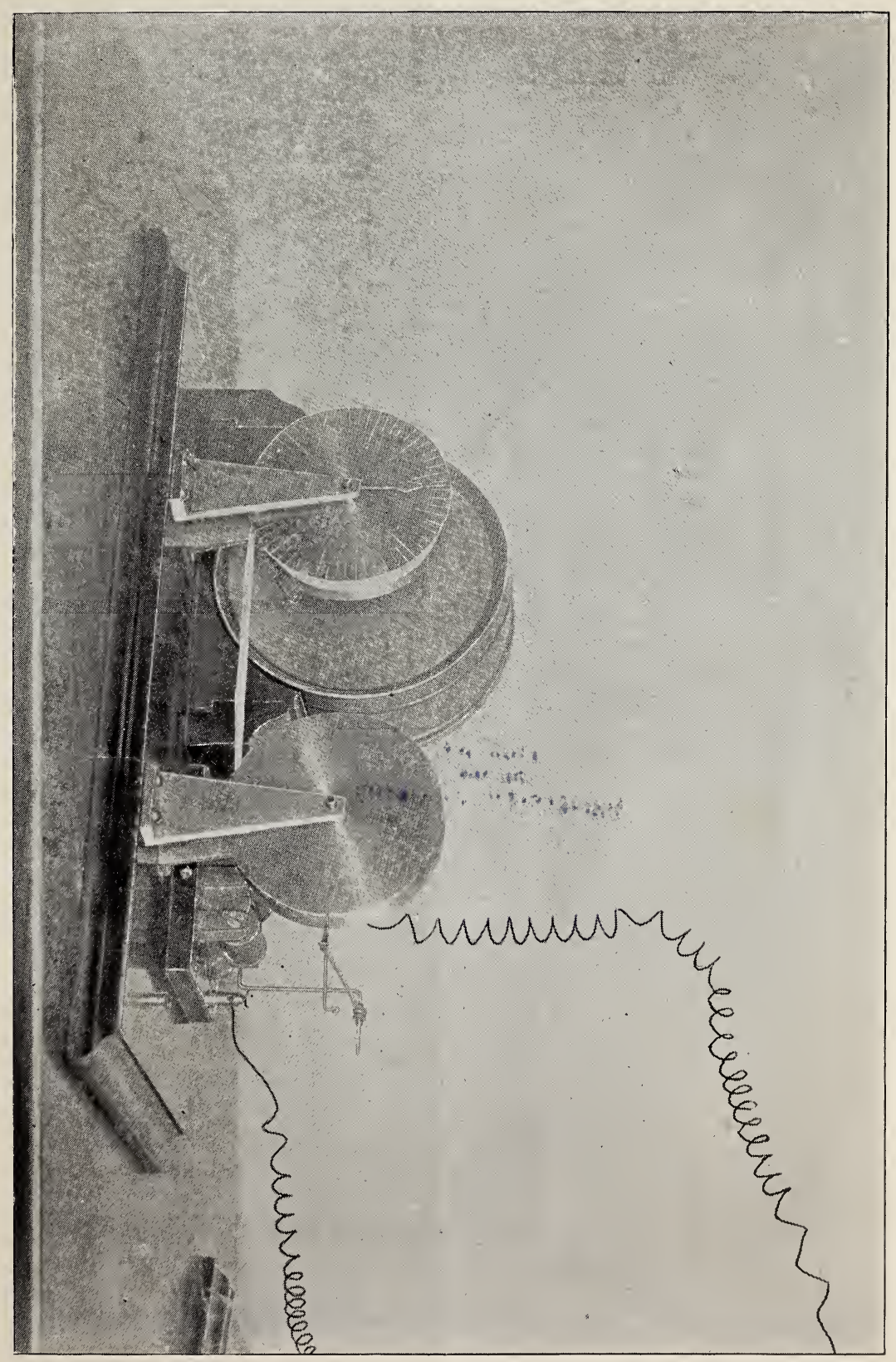

PLATE IX. 


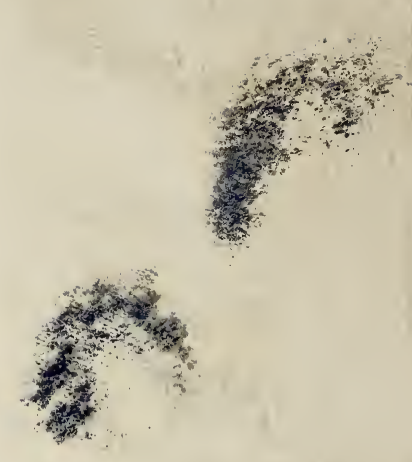

dienaris

of The

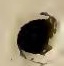


Bulletin No. 9. MINNESOTA BOTANICAL STUDIES. Sept 1894.

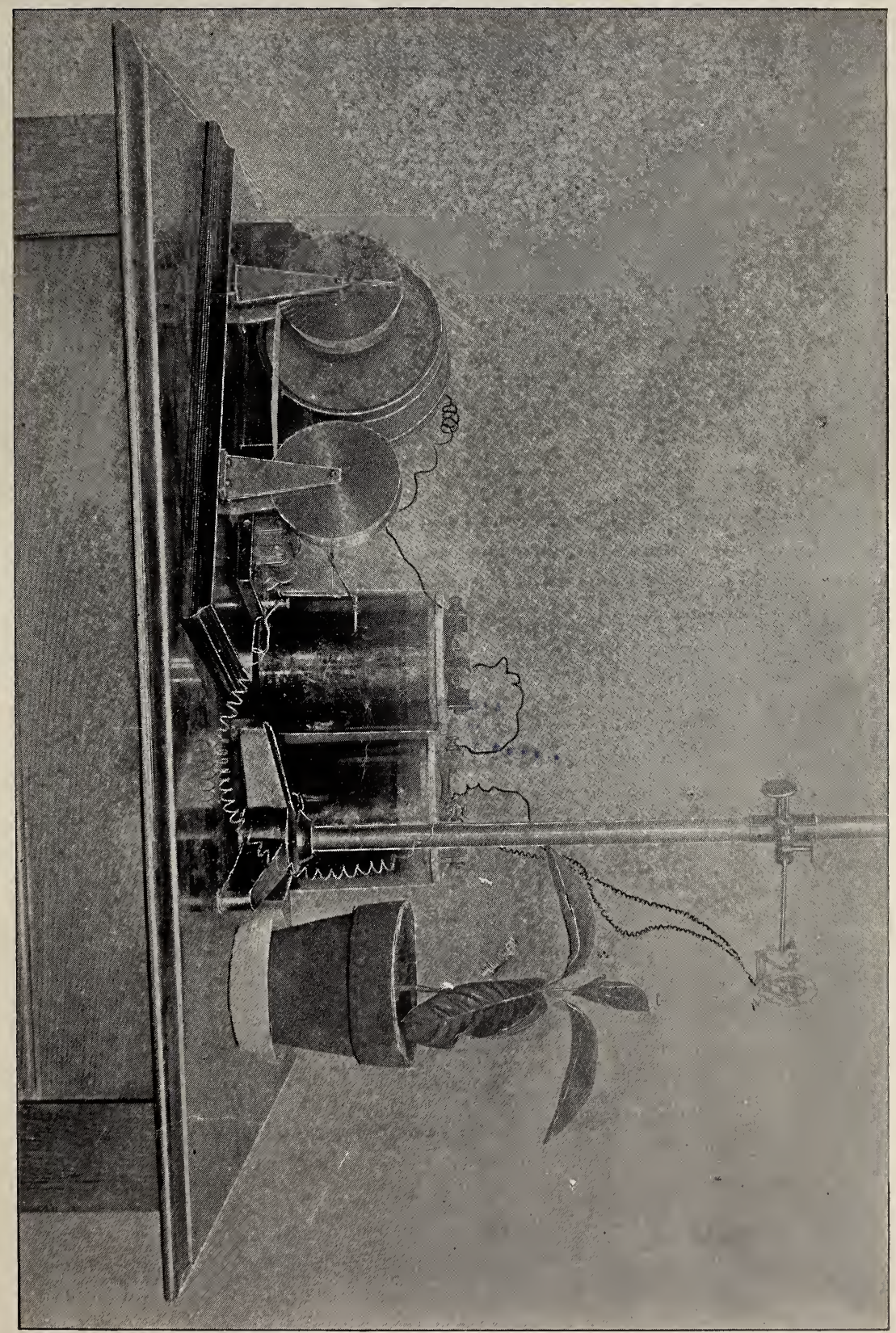

PLATE $X$. 


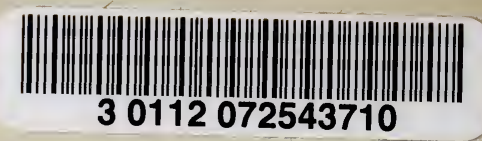

LIKRAPY
OF THE
UNIYERSIYY OF ILEINOIS

i.

* 